\title{
CONIC CONVEX PROGRAMMING AND SELF-DUAL EMBEDDING
}

\author{
Z.-Q. Luo ${ }^{1}$ and J.F. Sturm ${ }^{1}$ and S. Zhang ${ }^{2}$
}

April, 1998

\begin{abstract}
How to initialize an algorithm to solve an optimization problem is of great theoretical and practical importance. In the simplex method for linear programming this issue is resolved by either the two-phase approach or using the so-called big $M$ technique. In the interior point method, there is a more elegant way to deal with the initialization problem, viz. the self-dual embedding technique proposed by Ye, Todd and Mizuno [30]. For linear programming this technique makes it possible to identify an optimal solution or conclude the problem to be infeasible/unbounded by solving its embedded self-dual problem. The embedded self-dual problem has a trivial initial solution and has the same structure as the original problem. Hence, it eliminates the need to consider the initialization problem at all. In this paper, we extend this approach to solve general conic convex programming, including semidefinite programming. Since a nonlinear conic convex programming problem may lack the so-called strict complementarity property, it causes difficulties in identifying solutions for the original problem, based on solutions for the embedded self-dual system. We provide numerous examples from semidefinite programming to illustrate various possibilities which have no analogue in the linear programming case.
\end{abstract}

Key words: Self-duality, conic convex programming, semidefinite programming, initialization, interior point method.

AMS subject classification: 49M45, 90C25, 90C45.

${ }^{1}$ Communications Research Laboratory, McMaster University, Hamilton, Canada.

${ }^{2}$ Econometric Institute, Erasmus University Rotterdam, The Netherlands. 


\section{Introduction}

Like many other optimization methods, interior point methods are iterative in nature. That is, given an interior non-optimal solution, an interior point method would produce a sequence of improving solutions iteratively. Hence, an important question is: How can we get hold of an interior solution to activate the method? A similar question can be asked when one applies the simplex method to solve linear programming problems, because the simplex method assumes the availability of a feasible basic solution to start with. In that case, a common practice is to introduce an auxiliary problem for which a feasible basic solution is known and by solving the auxiliary problem, one can either conclude infeasibility, or indeed obtain a basic feasible solution of the original problem as desired. This is the well-known two-phase approach for the simplex method. A disadvantage of the two-phase approach is that in the feasibility phase, the objective function does not play any role, and consequently the feasible solution obtained may be far from any optimum. A remedy in this respect, is the so-called big $M$ method, which is basically a penalty approach trying to drive the infeasible elements out of the basis as soon as possible. Nevertheless, the big $M$ method works pretty much the same as the two-phase method, since it gives an absolute priority to the feasibility over the objective value.

The situation for the interior point methods is different. Not so long after the regular interior point methods for linear programming received intensive investigation, quest on the initialization issue led to the development of the so-called infeasible interior point methods; see Mizuno [14] for a survey. An infeasible interior point method starts with a possibly infeasible interior solution, and the iterates move towards an optimal solution of the original problem, if it exists, while reducing the feasibility residuals and the duality gap simultaneously. This technique was implemented in the highly successful interior point code OB1 of Lustig, Marsten and Shanno $[12,13]$ on the Netlib test problems [3]. However, when the original problem is either infeasible or unbounded, then infeasible interior point methods can have difficulty detecting these abnormalities. Later, an alternative, known as self-dual embedding, was introduced by Ye, Todd and Mizuno [30]. With the new technique, the original problem is nicely embedded together with its dual problem in a larger system. By solving the embedded system, the original problem is solved completely in the sense that if the problem is solvable, then an optimal solution is provided; if the problem is infeasible, then a Farkas type dual certificate is found; if the problem is unbounded, then an infinite-improving direction is established. So the new technique makes it a lot easier to detect infeasibility; see $\mathrm{Xu}$, Hung and Ye [28]. Moreover, the embedded system has a trivial initial interior feasible solution and its intrinsic size is precisely the same as the original problem. All these nice properties make clear that a usual path-following interior point method, in combination with the self-dual embedding technique, solves linear programming problems satisfactory. 
What remained as a point of research was to extend the self-dual embedding technique to solve optimization problems beyond the class of linear programming. Ye [29] showed that indeed the technique is also suitable for monotone LCPs. On one hand, this case is simpler than the linear programming case, since there are only two possible outcomes: either a complementary solution, or a certificate that no such solution exists. In particular, there is no distinction between primal and dual infeasibility. On the other hand, there is a nasty case here that requires two phases, viz. if the set of complementary solutions is unbounded and the so-called $q$-value is negative. This case cannot occur in linear programming, since the $q$-value of the associated complementarity systems is then known to be zero.

Another natural extension of linear programming is conic convex programming as discussed by Nesterov and Nemirovski [16]. A very important example of conic programming is semidefinite programming which has been the center of recent research activities in the interior point community. Several groups of authors independently extended the self-dual embedding technique to semidefinite programming, viz. Potra and Sheng [20], De Klerk, Roos and Terlaky [6], Luo, Sturm and Zhang [10] and Nesterov, Todd and Ye [19]. The latter two papers concern the more general case of conic convex programming, each with a different emphasis.

Nesterov, Todd and Ye [19] analyzed the application of logarithmically homogeneous barrier techniques to self-dual embeddings and considered the associated complexity issues. Luo, Sturm and Zhang [10] were concerned with a general duality theory for conic problems and with the question how to determine the status of the original problem from the sequence of iterates solving the selfdual embedding system. Report [10] was lengthy and covered two virtually separate topics. To make our results more accessible, we decide to split the main contents of [10] into two reports. The first one, [11], is completely devoted to duality theory. The second one is the current paper. In this part we will exclusively deal with the self-dual embedding techniques applied to the framework of conic programming. It turns out that a general conic programming problem may have subtle properties that a linear programming problem can never have. For example, it is possible that a conic programming problem with a finite optimal value does not have an attainable optimal solution, or an infeasible problem may become feasible by an infinitesimal change on the problem data. The goal of this paper is to discuss the self-dual embedding technique for conic problems in general, and the detection of the status of a given conic problem by means of solving the self-dual embedding system with a path-following interior point method in particular.

The organization of the paper is as follows. Section 2 presents a general introduction to conic convex programming problems. Sections 3 and 4 discuss schemes of self-dual embedding. Section 5 discusses how a weakly centered sequence can be used to solve a pure self-dual problem. In Section 7, the so-called self-dual embedding technique is discussed extensively. Various examples are given to illustrate how solutions/certificates for the original problem can be deduced from a weakly 
centered sequence for the embedded self-dual system. Discussions thus far depend crucially on the availability of a weakly centered sequence. The existence of such a sequence is shown in Section 8 . Finally, we conclude the paper in Section 9.

\section{Preliminaries}

We consider the conic convex program in the following form:

$$
\text { (P) } \quad p^{*}=\inf \left\{c^{\mathrm{T}} x \mid x \in(b+\mathcal{A}) \cap \mathcal{K}\right\}
$$

with its standard dual optimization program

$$
\text { (D) } d^{*}=\inf \left\{b^{T} z \mid z \in\left(c+\mathcal{A}^{\perp}\right) \cap \mathcal{K}^{*}\right\} \text {, }
$$

where $\mathcal{K} \subset \Re^{n}$ is a convex cone, $\mathcal{A}$ is a linear subspace of $\Re^{n}$ and $b$ and $c$ are given vectors in $\mathcal{A}^{\perp}$ and $\mathcal{A}$ respectively. The cone $\mathcal{K}^{*}$ is the dual cone associated with $\mathcal{K}$, which is defined as

$$
\mathcal{K}^{*}:=\left\{z \in \Re^{n} \mid x^{\mathrm{T}} z \geq 0 \forall x \in \mathcal{K}\right\}
$$

We assume that the convex cone $\mathcal{K}$ is closed, solid $($ int $\mathcal{K} \neq \emptyset)$ and pointed $(\mathcal{K} \cap-\mathcal{K}=\{0\})$. This assumption obviously holds true for the linear programming case, in which we deal with the nonnegative orthant $\mathcal{K}=\mathcal{K}^{*}=\Re^{n}$, and the semidefinite programming case, in which we deal with the cone of positive semidefinite matrices $\mathcal{K}=\mathcal{K}^{*}=\{X \mid X \succeq 0\}$. We remark that our assumptions on $\mathcal{K}$ imply that the dual cone $\mathcal{K}^{*}$ is also closed, solid and pointed.

When we solve $(\mathrm{P})$, we intend to find an optimal solution and its optimality certificate if such ones exist, and if not, we shall prove the fact that no optimal solution exists also by a certificate. Solutions of (D) play the role of certificates. For primal optimality a dual optimal solution is a certificate, and for primal infeasibility a Farkas type dual direction is a certificate. Therefore, the primal problem (P) and its dual (D) are closely linked, and are often being solved together. This interpretation of duality is well known for linear programming, and is widely used in various algorithmic approaches.

The homogeneous self-dual model of Goldman and Tucker combines primal solutions and both types of dual solutions in a single model. Unfortunately, the homogeneous self-dual model does not

only include certified solutions. In particular, the origin is trivially a solution to any homogeneous self-dual model. So, not all solutions to the homogeneous self-dual model are of interest.

But, if a solution to the homogenous self-dual model is strictly complementary, then it does consist of a certified solution, except for one case. The exceptional case is due to the primal-dual symmetry: 
the strictly complementary solution may yield a Farkas-type proof of dual infeasibility, in which case we have still to decide whether the primal is infeasible or unbounded.

The main reason why we can use the nice properties of the self-dual model is that almost all algorithms in the class of interior point methods converge to a strictly complementary solution, provided that such a solution exists. This useful property of interior point methods was made clear in Güler and Ye [5]. Unfortunately, the interior point method cannot be applied directly to the homogeneous self-dual model, since this model admits no interior solutions. Therefore, Ye, Todd and Mizuno [30] embedded the homogeneous self-dual model into an extended self-dual program which has one extra variable and constraint, and a trivial, perfectly centered interior solution.

In this paper we will extend the homogenous self-dual model and its embedding to include the conic programs (P) and (D). Much remains the same as in the linear porgramming case. Complications arise in the boundary cases. To be specific, we consider $(\mathrm{P})$ for instance. We call $(\mathrm{P})$ infeasible if the set $(b+\mathcal{A}) \cap \mathcal{K}$ is empty. Still, in that case there are two possibilities in general, namely $\operatorname{dist}(b+\mathcal{A}, \mathcal{K})>0$ or $\operatorname{dist}(b+\mathcal{A}, \mathcal{K})=0$. If $\operatorname{dist}(b+\mathcal{A}, \mathcal{K})>0$ we call $(\mathrm{P})$ strongly infeasible, and if $\operatorname{dist}(b+\mathcal{A}, \mathcal{K})=0$ we call $(\mathrm{P})$ weakly infeasible. Remark that weakly infeasibility is impossible in linear programming.

Note that if $(\mathrm{P})$ is weakly infeasible then there exists $x^{(1)}, x^{(2)}, \ldots$, such that

$$
\operatorname{dist}\left(x^{(k)}, b+\mathcal{A}\right)+\operatorname{dist}\left(x^{(k)}, \mathcal{K}\right) \rightarrow 0 .
$$

It is now interesting to introduce the subvalue $p^{-}$defined as

$$
p^{-}:=\lim _{\epsilon \downarrow 0} \inf _{x}\left\{c^{\mathrm{T}} x \mid \operatorname{dist}(x, b+\mathcal{A})+\operatorname{dist}(x, \mathcal{K})<\epsilon\right\} .
$$

If $(\mathrm{P})$ is infeasible then obviously $p^{*}=\infty$, but the subvalue $p^{-}$may be finite (or even $-\infty$ ) if it is only weakly infeasible. Examples of such phenomena can be found in Section 6.1.

The subvalue $p^{-}$is closely related to the dual optimal value $d^{*}$ in the following way:

$$
d^{*}= \begin{cases}p^{-}=\infty, & \text { if }(\mathrm{D}) \text { infeasible and }(\mathrm{P}) \text { strongly infeasible } \\ -p^{-}, & \text {otherwise }\end{cases}
$$

(see $[11,23])$. Similarly, the dual subvalue $d^{-}$can be defined.

General conic duality relation resembles the linear programming case very well if Slater's condition holds, i.e. if $(b+\mathcal{A}) \cap$ int $\mathcal{K} \neq \emptyset$. Under Slater's condition we have

$$
p^{*}=p^{-}=-d^{*} .
$$

Moreover, if $p^{*}>-\infty$ then the dual optimum is attained, and the set of dual optimal solutions is bounded. 
The above discussed duality provides a means to certify optimality. Farkas-type duality provides a means to certify infeasibility. A Farkas-type dual solution, which we usually call a dual improving direction, is a vector $z \in \mathcal{A}^{\perp} \cap \mathcal{K}^{*}$ with $b^{\mathrm{T}} z<0$. Such a solution exists if and only if the primal is strongly infeasible.

Notice however that there exists no dual improving direction if $(\mathrm{P})$ is only weakly infeasible. So, how can we certify infeasibility if $(\mathrm{P})$ is only weakly infeasible? For this, we need the concept of a dual improving direction sequence, which is a sequence $z^{(1)}, z^{(2)}, \ldots$ such that

$$
\limsup _{k \rightarrow \infty} b^{\mathrm{T}} z^{(k)}<0, \quad \lim _{k \rightarrow \infty}\left(\operatorname{dist}\left(z^{(k)}, \mathcal{A}^{\perp}\right)+\operatorname{dist}\left(z^{(k)}, \mathcal{K}^{*}\right)\right)=0 .
$$

Programs that have interior solutions, i.e. $(b+\mathcal{A}) \cap$ int $\mathcal{K} \neq \emptyset$, are said to be strongly feasible. We have already remarked that if $(\mathrm{P})$ is strongly feasible, then $p^{*}=p^{-}$. It is possible to certify that a problem is not strongly feasible, by using yet another type of dual solutions: nonzero dual lower level directions. A nonzero dual lower level direction is by definition a vector $0 \neq z \in \mathcal{A}^{\perp} \cap \mathcal{K}^{*}$ such that $b^{\mathrm{T}} z \leq 0$. Such directions exist if and only if $(\mathrm{P})$ has no interior feasible solutions $[11,23]$.

For later use, we list here two general rules for dualizing cones. First, for any convex cone $\mathcal{K} \subseteq \Re^{n}$ and invertible matrix $M \in \Re^{n \times n}$, it holds that

$$
\left(M^{\mathrm{T}} \mathcal{K}\right)^{*}=M^{-1} \mathcal{K}^{*}
$$

see $[11,23]$. Second, for two convex cones $\mathcal{K}_{1}$ and $\mathcal{K}_{2}$, it holds that

$$
\mathcal{K}_{1}^{*} \cap \mathcal{K}_{2}^{*}=\left(\mathcal{K}_{1}+\mathcal{K}_{2}\right)^{*}
$$

The well known bipolar theorem povides a dual characterization of closed convex cones, viz.

$$
\mathcal{K}=\left\{x \in \Re^{n} \mid x^{\mathrm{T}} y \geq 0 \forall y \in \mathcal{K}^{*}\right\} .
$$

Similarly, the interior of a solid convex cone is characterized by

$$
\text { int } \mathcal{K}=\left\{x \in \Re^{n} \mid x^{\mathrm{T}} y>0 \forall 0 \neq y \in \mathcal{K}^{*}\right\} \text {. }
$$

\section{$3 \quad$ Self-Duality}

Self-duality has been defined by Duffin [2] for conic convex programs that are formulated in the so-called symmetric form. More recently, Ye, Todd and Mizuno [30] formulated a linear program in a different form, and argued that their program is self-dual since "the dual of the problem is equivalent to the primal". Below, we propose a definition of self-duality that does not depend on the specific form in which the program is formulated. 
Definition 1 A conic convex program $C P(b, c, \mathcal{A}, \mathcal{K})$ is $\Pi$ self-dual if $\Pi$ is a symmetric permutation matrix such that

$$
c=\Pi b, \mathcal{A}^{\perp}=\Pi \mathcal{A}, \mathcal{K}^{*}=\Pi \mathcal{K} .
$$

If, in addition, $b=c=0$, then the program is said to be homogeneous.

Notice from the above definition that a self-dual program is indeed its own dual, after a simple reordering of the variables. More precisely, if $\mathrm{CP}(b, c, \mathcal{A}, \mathcal{K})$ is a $\Pi$ self-dual conic convex program, then

$$
\Pi(b+\mathcal{A})=c+\mathcal{A}^{\perp}, \quad \Pi \mathcal{K}=\mathcal{K}^{*},
$$

so that $x \in \Re^{n}$ is primal feasible if and only if $\Pi x$ is dual feasible, and, using the symmetry of $\Pi$,

$$
c^{\mathrm{T}}=b^{\mathrm{T}} \Pi,
$$

so that the primal objective value $c^{\mathrm{T}} x$ is identical to the dual objective value $b^{\mathrm{T}}(\Pi x)$ for all $x \in \Re^{n}$. Notice also that $\mathcal{K}^{*}=\Pi \mathcal{K}$ implies that $\mathcal{K}$ is closed; self-dual conic convex programs are therefore always closed.

Remark 1 The requirement that $\Pi$ is symmetric, i.e. $\Pi=\Pi^{T}$, is natural. Namely, for any permutation matrix $\Pi$, there holds $\Pi^{T}=\Pi^{-1}$. From the relation $\mathcal{A}^{\perp}=\Pi \mathcal{A}$, it therefore follows using (3) that

$$
\Pi \mathcal{A}=\mathcal{A}^{\perp}=\left(\Pi^{T} \Pi \mathcal{A}\right)^{\perp}=\Pi^{-1}(\Pi \mathcal{A})^{\perp}=\Pi^{T} \mathcal{A},
$$

i.e. $\Pi \mathcal{A}=\Pi^{T} \mathcal{A}$ and similarly, $\Pi \mathcal{A}^{\perp}=\Pi^{T} \mathcal{A}^{\perp}$. Hence, the relation $\mathcal{A}^{\perp}=\Pi \mathcal{A}$ already implies some symmetry.

Below are two elementary results for self-dual conic convex programs.

Lemma 1 Let $\mathcal{A}$ be a linear subspace of $\Re^{n}$ such that $\mathcal{A}^{\perp}=\Pi \mathcal{A}$ for some symmetric permutation matrix $\Pi$. Then

$$
\Pi P_{\mathcal{A}}=P_{\mathcal{A}^{\perp}} \Pi, \quad \Pi P_{\mathcal{A}^{\perp}}=P_{\mathcal{A}} \Pi
$$

Proof. Since $\Pi$ is a symmetric permutation matrix, we know that $\Pi \mathcal{A}^{\perp}=\Pi^{2} \mathcal{A}=\mathcal{A}$. Hence, the orthogonal decomposition

$$
x=y+z, \quad y \in \mathcal{A}, z \in \mathcal{A}^{\perp}
$$

is equivalent to

$$
\Pi x=\Pi y+\Pi z, \quad \Pi y \in \mathcal{A}^{\perp}, \Pi z \in \mathcal{A} .
$$


From the above decompositions, it follows for arbitrary $x$ that $\Pi P_{\mathcal{A}} x=\Pi y$ and $P_{\mathcal{A}^{\perp}} \Pi x=\Pi y$ respectively.

Lemma 2 Let $C P(b, c, \mathcal{A}, \mathcal{K})$ be a $\Pi$ self-dual program where $\mathcal{K}$ is a solid convex cone. Then

$$
y^{T} P_{\mathcal{A}} \Pi y=y^{T} P_{\mathcal{A}^{\perp}} \Pi y=\frac{1}{2} y^{T} \Pi y>0 \text { for all } y \in \text { int } \mathcal{K} .
$$

Proof. Let $y \in$ int $\mathcal{K}$, then

$$
\Pi y \in \Pi \operatorname{int} \mathcal{K}=\operatorname{int} \Pi \mathcal{K}=\operatorname{int} \mathcal{K}^{*}
$$

where in the first identity, we used the fact that $\Pi$ is an invertible matrix. Since $\mathcal{K}^{*}$ is pointed, and $0 \neq \Pi y \in$ int $\mathcal{K}^{*}$, it follows from (5) that

$$
0<y^{\mathrm{T}} \Pi y=y^{\mathrm{T}} \Pi P_{\mathcal{A}} y+y^{\mathrm{T}} \Pi P_{\mathcal{A}^{\perp}} y .
$$

Using now Lemma 1 and the symmetricity of $\Pi$, the lemma follows.

The properties of self-dual conic convex programs result in a particularly nice form of weak-duality:

Theorem 1 If $C P(b, c, \mathcal{A}, \mathcal{K})$ is a $\Pi$ self-dual conic convex program, then

$$
c^{T} x=\frac{1}{2} x^{T} \Pi x \geq 0
$$

for all $x \in(b+\mathcal{A}) \cap \mathcal{K}$.

Proof. Since $x-b \in \mathcal{A}, \Pi x-c \in \mathcal{A}^{\perp}$ and $b \perp c$, we have

$$
0=(x-b)^{\mathrm{T}}(\Pi x-c)=x^{\mathrm{T}} \Pi x-b^{\mathrm{T}} \Pi x-c^{\mathrm{T}} x=x^{\mathrm{T}} \Pi x-2 c^{\mathrm{T}} x .
$$

Moreover, $x^{\mathrm{T}} \Pi x \geq 0$ because $x \in \mathcal{K}$ and $\Pi x \in \mathcal{K}^{*}$.

If $x^{*}$ is a solution to a $\Pi$ self-dual program $\operatorname{CP}(b, c, \mathcal{A}, \mathcal{K})$ and $\left(x^{*}\right)^{\mathrm{T}} \Pi x^{*}=0$, then $x^{*}$ is called a self-complementary solution. It follows from Theorem 1 that if $x^{*}$ is a self-complementary solution, then it is also an optimal solution.

As before, we denote the optimal value and the subvalue of the conic convex program $\mathrm{CP}(b, c, \mathcal{A}, \mathcal{K})$ by $p^{*}$ and $p^{-}$, respectively. That is,

$$
p^{*}:=\inf _{x}\left\{c^{\mathrm{T}} x \mid x \in(b+\mathcal{A}) \cap \mathcal{K}\right\}, \quad p^{-}:=\liminf _{\epsilon \downarrow 0} \inf _{x}\left\{c^{\mathrm{T}} x \mid x \in \mathcal{K}, \operatorname{dist}(x, b+\mathcal{A})<\epsilon\right\} .
$$


If $\operatorname{CP}(b, c, \mathcal{A}, \mathcal{K})$ is self-dual, then

$$
p^{-}=-p^{*},
$$

as follows from (1). Since $p^{*} \geq p^{-}$, we know that the optimal value $p^{*}$ is nonnegative, and the subvalue $p^{-}$is non-positive. This implies in particular that a self-dual program cannot be unbounded.

\section{Self-Dual Embedding}

There are basically two different types of dual variables involved in closed conic convex programming, viz.

- Dual feasible solutions, and

- Nonzero dual directions.

The former yield lower bounds on the optimal value, and the latter concern the feasibility of the problem. The homogeneous self-dual embedding, to be discussed in Section 4.1, combines both types of dual variables into a single self-dual program. In Section 4.2, we will treat the extended self-dual model, which is a strongly feasible self-dual model for which the optimal solution set corresponds to feasible solutions of the homogeneous self-dual model.

\subsection{The Homogeneous Self-Dual Model}

Consider a $\Pi_{\mathrm{SD}}$ self-dual program (SD),

$$
\inf _{x_{\mathrm{SD}}}\left\{c_{\mathrm{SD}}^{\mathrm{T}} x_{\mathrm{SD}} \mid x_{\mathrm{SD}} \in\left(b_{\mathrm{SD}}+\mathcal{A}_{\mathrm{SD}}\right) \cap \mathcal{K}_{\mathrm{SD}}\right\}
$$

i.e. $(\mathrm{SD})$ is the conic convex program $\mathrm{CP}\left(b_{\mathrm{SD}}, c_{\mathrm{SD}}, \mathcal{A}_{\mathrm{SD}}, \mathcal{K}_{\mathrm{SD}}\right)$. We assume that $\mathcal{K}_{\mathrm{SD}}$ is solid. The optimal value of (SD) is denoted by $p_{\mathrm{SD}}^{*}$. We introduce an invertible matrix $M\left(b_{\mathrm{SD}}, c_{\mathrm{SD}}\right)$,

$$
M\left(b_{\mathrm{SD}}, c_{\mathrm{SD}}\right):=\left[\begin{array}{ccc}
I & b_{\mathrm{SD}} & 0 \\
0 & 1 & 0 \\
-c_{\mathrm{SD}}^{\mathrm{T}} & 0 & 1
\end{array}\right],
$$

and we let

$$
\mathcal{A}_{\mathrm{H}}:=M\left(b_{\mathrm{SD}}, c_{\mathrm{SD}}\right)\left(\mathcal{A}_{\mathrm{SD}} \times \Re \times\{0\}\right), \quad \mathcal{K}_{\mathrm{H}}:=\mathcal{K}_{\mathrm{SD}} \times \Re_{+} \times \Re_{+} .
$$

Applying (3), we have

$$
\mathcal{A}_{\mathrm{H}}^{\perp}=M\left(b_{\mathrm{SD}}, c_{\mathrm{SD}}\right)^{-\mathrm{T}}\left(\mathcal{A}_{\mathrm{SD}}^{\perp} \times\{0\} \times \Re\right),
$$


where

$$
M\left(b_{\mathrm{SD}}, c_{\mathrm{SD}}\right)^{-\mathrm{T}}=\left[\begin{array}{ccc}
I & 0 & c_{\mathrm{SD}} \\
-b_{\mathrm{SD}}^{\mathrm{T}} & 1 & 0 \\
0 & 0 & 1
\end{array}\right] .
$$

Using the self-duality of (SD), it follows that $\mathrm{CP}\left(0,0, \mathcal{A}_{\mathrm{H}}, \mathcal{K}_{\mathrm{H}}\right)$ is a homogeneous $\Pi_{\mathrm{H}}$ self-dual program with

$$
\Pi_{\mathrm{H}}=\left[\begin{array}{ccc}
\Pi_{\mathrm{SD}} & 0 & 0 \\
0 & 0 & 1 \\
0 & 1 & 0
\end{array}\right]
$$

This type of homogeneous self-dual program was proposed by Goldman and Tucker $[4,26]$ in the context of linear programming. The convex cone $\mathcal{A}_{\mathrm{H}} \cap \mathcal{K}_{\mathrm{H}}$ consists of those solutions $\left(x_{\mathrm{SD}}, x_{0}, z_{0}\right)$ for which

$$
\left\{\begin{array}{l}
x_{\mathrm{SD}} \in\left(x_{0} b_{\mathrm{SD}}+\mathcal{A}_{\mathrm{SD}}\right) \cap \mathcal{K}_{\mathrm{SD}} \\
x_{0} \geq 0 \\
z_{0}=-c_{\mathrm{SD}}^{\mathrm{T}} x_{\mathrm{SD}} \geq 0
\end{array}\right.
$$

The concepts of complementary solution, improving direction and nonzero lower level direction for (SD) can be characterized in terms of the homogeneous program $\left(0,0, \mathcal{A}_{\mathrm{H}}, \mathcal{K}_{\mathrm{H}}\right)$ as follows:

- $x_{\mathrm{SD}}$ is a self-complementary solution to (SD) if and only if

$$
\left(x_{\mathrm{SD}}, 1,0\right) \in \mathcal{A}_{\mathrm{H}} \cap \mathcal{K}_{\mathrm{H}} .
$$

- $x_{\mathrm{SD}}$ is an improving direction for (SD) if and only if

$$
\left(x_{\mathrm{SD}}, 0, z_{0}\right) \in \mathcal{A}_{\mathrm{H}} \cap \mathcal{K}_{\mathrm{H}} \text { for some } z_{0}>0 .
$$

- $x_{\mathrm{SD}}$ is a nonzero lower level direction for (SD) if and only if

$$
\left(x_{\mathrm{SD}}, 0, z_{0}\right) \in \mathcal{A}_{\mathrm{H}} \cap \mathcal{K}_{\mathrm{H}} \backslash\{0\} \text { for some } z_{0} \geq 0 \text {. }
$$

With the above observation, it is straightforward to prove the following theorem.

Theorem 2 If $\left(x_{S D}, x_{0}, z_{0}\right) \in \mathcal{A}_{H} \cap \mathcal{K}_{H}$, then

$$
x_{S D}^{T} \Pi_{S D} x_{S D}=0, \quad x_{0} z_{0}=0 .
$$

Moreover, if $\left(x_{S D}, x_{0}, z_{0}\right) \in \mathcal{A}_{H} \cap \mathcal{K}_{H} \backslash\{0\}$ and $(S D)$ is strongly feasible, then $x_{0}>0$ and $x_{S D} / x_{0}$ is a self-complementary solution of $(S D)$. 
Remark 2 It follows from Theorem 2 that if

$$
\left(x_{S D}, 0, z_{0}\right) \in \mathcal{A}_{H} \cap \mathcal{K}_{H} \backslash\{0\}
$$

then (SD) is not strongly feasible, i.e. it is either weakly feasible, or weakly infeasible, or strongly infeasible. However, if it is strongly infeasible, then it must have an improving direction $x_{S D}^{\prime}$ and $\left(x_{S D}^{\prime}, 0,-c_{S D}^{T} x_{S D}^{\prime}\right) \in \mathcal{A}_{H} \cap \mathcal{K}_{H}$.

Remark 3 Even if $(S D)$ is not strongly feasible, it may have a self-complementary solution $x_{S D}$, in which case $\left(x_{S D}, 1,0\right) \in \mathcal{A}_{H} \cap \mathcal{K}_{H}$.

\subsection{The Extended Self-Dual Model}

Given a $\Pi_{\mathrm{SD}}$ self-dual program (SD), we have constructed a $\Pi_{\mathrm{H}}$ homogeneous self-dual model $\left(0,0, \mathcal{A}_{\mathrm{H}}, \mathcal{K}_{\mathrm{H}}\right)$, generalizing the Goldman-Tucker [4] model to conic convex programming. We will now add a normalization constraint to the model, and we will make the program strongly dual. This results in an extension of Ye, Todd and Mizuno's self-dual formulation [30] for linear programming, to the context of conic convex programming.

Choose $\iota \in$ int $\mathcal{K}_{\mathrm{H}}$, and let

$$
\rho:=\frac{\iota^{\mathrm{T}} P_{\mathcal{A}_{\mathrm{H}}} \Pi_{\mathrm{H}} \iota}{\left\|P_{\mathcal{A}_{\mathrm{H}}^{\perp} \iota}\right\|_{2}^{2}} .
$$

By construction, $\mathcal{K}_{\mathrm{H}}$ is solid, and we can apply Lemma 2 to conclude that $\rho$ is a well defined positive quantity. Define

$$
b_{\mathrm{E}}:=\rho P_{\mathcal{A}_{\mathrm{H}}} \Pi_{\mathrm{H}} \iota, \quad c_{\mathrm{E}}:=\rho P_{\mathcal{A}_{\mathrm{H}}^{\perp} \iota},
$$

and notice from Lemma 1 that

$$
c_{\mathrm{E}}=\Pi_{\mathrm{H}} b_{\mathrm{E}} .
$$

Since $c_{\mathrm{E}}$ is simply a permutation of $b_{\mathrm{E}}$ and $\rho>0$, we have

$$
\left\|b_{\mathrm{E}}\right\|_{2}=\left\|c_{\mathrm{E}}\right\|_{2}>0
$$

Using the definitions of $c_{\mathrm{E}}$ and $\rho$, and then applying Lemma 2, we further have

$$
\frac{\left\|b_{\mathrm{E}}\right\|_{2}^{2}}{\rho}=\frac{\left\|c_{\mathrm{E}}\right\|_{2}^{2}}{\rho}=\rho\left\|P_{\mathcal{A}_{\mathrm{H}}^{\perp}} \iota\right\|_{2}^{2}=\iota^{\mathrm{T}} P_{\mathcal{A}_{\mathrm{H}}} \Pi_{\mathrm{H}} \iota=\frac{\iota^{\mathrm{T}} \Pi_{\mathrm{H}} \iota}{2} .
$$

We shall now study the conic convex program $\mathrm{CP}\left(b_{\mathrm{E}}, c_{\mathrm{E}}, \mathcal{A}_{\mathrm{E}}, \mathcal{K}_{\mathrm{H}}\right)$, where

$$
\mathcal{A}_{\mathrm{E}}:=\left(\mathcal{A}_{\mathrm{H}} \cap \operatorname{Ker} b_{\mathrm{E}}^{\mathrm{T}}\right)+\operatorname{Img} c_{\mathrm{E}} \text {. }
$$


Just as in the homogeneous self-dual model, we partition the decision variable as

$$
x_{\mathrm{E}}=\left(x_{\mathrm{SD}}, x_{0}, z_{0}\right),
$$

with $x_{\mathrm{SD}} \in \mathcal{K}_{\mathrm{SD}}, x_{0} \in \Re_{+}$and $z_{0} \in \Re_{+}$. Introducing an auxiliary variable

$$
y_{0}:=\frac{\rho}{\left\|c_{\mathrm{E}}\right\|_{2}^{2}} c_{\mathrm{E}}^{\mathrm{T}} x_{\mathrm{E}},
$$

we can reformulate $\mathrm{CP}\left(b_{\mathrm{E}}, c_{\mathrm{E}}, \mathcal{A}_{\mathrm{E}}, \mathcal{K}_{\mathrm{H}}\right)$ as follows:

$$
\begin{array}{ll}
\min & \left(\iota^{\mathrm{T}} \Pi_{\mathrm{H}} \iota / 2\right) y_{0} \\
\text { s.t. } & x_{\mathrm{E}}-y_{0} \iota \in \mathcal{A}_{\mathrm{H}} \\
& b_{\mathrm{E}}^{\mathrm{T}} x_{\mathrm{E}}=\left\|b_{\mathrm{E}}\right\|_{2}^{2} \\
& x_{\mathrm{E}} \in \mathcal{K}_{\mathrm{H}}, y_{0} \in \Re .
\end{array}
$$

To see this, remark that by definition of $c_{\mathrm{E}}$,

$$
x_{\mathrm{E}}-y_{0} \iota \in \mathcal{A}_{\mathrm{H}} \Longleftrightarrow x_{\mathrm{E}}-\frac{y_{0}}{\rho} c_{\mathrm{E}} \in \mathcal{A}_{\mathrm{H}} .
$$

Since obviously,

$$
b_{\mathrm{E}}^{\mathrm{T}} x_{\mathrm{E}}=\left\|b_{\mathrm{E}}\right\|_{2}^{2} \Longleftrightarrow x_{\mathrm{E}}-b_{\mathrm{E}} \in \operatorname{Ker} b_{\mathrm{E}}^{\mathrm{T}},
$$

we obtain from the above two relations and the fact that $b_{\mathrm{E}} \in \mathcal{A}_{\mathrm{H}}, c_{\mathrm{E}} \in \mathcal{A}_{\mathrm{H}}^{\perp}$, that

$$
x_{\mathrm{E}}-b_{\mathrm{E}} \in \mathcal{A}_{\mathrm{E}} \Longleftrightarrow\left\{\begin{array}{l}
x_{\mathrm{E}}-y_{0} \iota \in \mathcal{A}_{\mathrm{H}}, \\
b_{\mathrm{E}}^{\mathrm{T}} x_{\mathrm{E}}=\left\|b_{\mathrm{E}}\right\|_{2}^{2}
\end{array}\right.
$$

From the relation $x_{\mathrm{E}} \in y_{0} \iota+\mathcal{A}_{\mathrm{H}}$, we obtain

$$
\iota^{\mathrm{T}} \Pi_{\mathrm{H}}\left(P_{\mathcal{A}_{\mathrm{H}}^{\perp}} x_{\mathrm{E}}\right)=\iota^{\mathrm{T}} \Pi_{\mathrm{H}}\left(y_{0} P_{\mathcal{A}_{\mathrm{H}}^{\perp}} \iota\right)=\frac{y_{0}}{2} \iota^{\mathrm{T}} \Pi_{\mathrm{H}} \iota,
$$

where we used Lemma 2 in the last identity. Using the definition of $b_{\mathrm{E}}$, it thus follows that

$$
\frac{b_{\mathrm{E}}^{\mathrm{T}} x_{\mathrm{E}}}{\rho}=\iota^{\mathrm{T}} \Pi_{\mathrm{H}} x_{\mathrm{E}}-\frac{y_{0}}{2} \iota^{\mathrm{T}} \Pi_{\mathrm{H}} \iota .
$$

Combining this with (9), we obtain an alternative form for the normalization constraint of (E), viz.

$$
b_{\mathrm{E}}^{\mathrm{T}} x_{\mathrm{E}}=\left\|b_{\mathrm{E}}\right\|_{2}^{2} \Longleftrightarrow \iota^{\mathrm{T}} \Pi_{\mathrm{H}} x_{\mathrm{E}}=\frac{1+y_{0}}{2} \iota^{\mathrm{T}} \Pi_{\mathrm{H}} \iota .
$$

It is obvious from (11) that the lower level sets of (E) are bounded. Moreover, it is now easily verified that $\iota \in\left(b_{\mathrm{E}}+\mathcal{A}_{\mathrm{E}}\right) \cap$ int $\mathcal{K}_{\mathrm{H}}$, i.e. $\iota$ is an interior solution, which can serve as an initial solution in interior point methods (remark that we can choose any $\iota \in$ int $\mathcal{K}_{\mathrm{H}}$ ). The feasible solutions $x_{\mathrm{E}}$ of $(\mathrm{E})$ for which $y_{0}=0$ correspond to those solutions of the homogeneous model $(\mathrm{H})$ that are normalized by the constraint $\iota^{\mathrm{T}} \Pi_{\mathrm{H}} x_{\mathrm{E}}=\iota^{\mathrm{T}} \Pi_{\mathrm{H}} \iota / 2$. The normalization guarantees that if $y_{0}=0$, then $x_{\mathrm{E}}$ is a nonzero direction of the homogeneous model $(\mathrm{H})$. 
Theorem 3 The conic convex program $C P\left(b_{E}, c_{E}, \mathcal{A}_{E}, \mathcal{K}_{H}\right)$ has the following properties:

1. It is self-dual,

2. It has an interior solution, viz. $\iota \in\left(b_{E}+\mathcal{A}_{E}\right) \cap$ int $\mathcal{K}_{H}$, and hence

3. It has a self-complementary solution,

4. Any self-complementary solution is a nonzero direction of $C P\left(0,0, \mathcal{A}_{H}, \mathcal{K}_{H}\right)$, and

5. For any nonzero direction $x_{E}$ of the homogeneous program $C P\left(0,0, \mathcal{A}_{H}, \mathcal{K}_{H}\right)$, there exists $\alpha>0$ such that $\alpha x_{S D}$ is a self-complementary solution for $C P\left(b_{E}, c_{E}, \mathcal{A}_{E}, \mathcal{K}_{H}\right)$.

\section{Proof.}

1. It is already known from (8) that $c_{\mathrm{E}}=\Pi_{\mathrm{H}} b_{\mathrm{E}}$. Moreover, $\mathcal{K}_{\mathrm{H}}^{*}=\Pi_{\mathrm{H}} \mathcal{K}_{\mathrm{H}}$, since $\mathrm{CP}\left(0,0, \mathcal{A}_{\mathrm{H}}, \mathcal{K}_{\mathrm{H}}\right)$ is self-dual. It remains to show that $\mathcal{A}_{\mathrm{E}}^{\perp}=\Pi_{\mathrm{H}} \mathcal{A}_{\mathrm{E}}$. To this end, we remark using (4) that

$$
\begin{aligned}
\mathcal{A}_{\mathrm{E}}^{\perp} & =\left[\left(\mathcal{A}_{\mathrm{H}} \cap \operatorname{Ker} b_{\mathrm{E}}^{\mathrm{T}}\right)+\operatorname{Img} c_{\mathrm{E}}\right]^{\perp} \\
& =\left(\mathcal{A}_{\mathrm{H}} \cap \operatorname{Ker} b_{\mathrm{E}}^{\mathrm{T}}\right)^{\perp} \cap \operatorname{Ker} c_{\mathrm{E}}^{\mathrm{T}} \\
& =\left(\mathcal{A}_{\mathrm{H}}^{\perp}+\operatorname{Img} b_{\mathrm{E}}\right) \cap \operatorname{Ker} c_{\mathrm{E}}^{\mathrm{T}} \\
& =\left(\mathcal{A}_{\mathrm{H}}^{\perp} \cap \operatorname{Ker} c_{\mathrm{E}}^{\mathrm{T}}\right)+\operatorname{Img} b_{\mathrm{E}},
\end{aligned}
$$

where we used $b_{\mathrm{E}} \perp c_{\mathrm{E}}$ in the last identity. Using (8) and the fact that $\mathcal{A}_{\mathrm{H}}^{\perp}=\Pi_{\mathrm{H}} \mathcal{A}_{\mathrm{H}}$, it follows that $\mathcal{A}_{\mathrm{E}}^{\perp}=\Pi_{\mathrm{H}} \mathcal{A}_{\mathrm{E}}$.

2. Using (11), it is easily verified that the solution $x_{\mathrm{E}}=\iota, y_{0}=1$ satisfies all the constraints of $(\mathrm{E})$.

3. The self-dual program $\mathrm{CP}\left(b_{\mathrm{E}}, c_{\mathrm{E}}, \mathcal{A}_{\mathrm{E}}, \mathcal{K}_{\mathrm{H}}\right)$ has a self-complementary solution because it is strongly feasible, see (2).

4. Let $x_{\mathrm{E}}$ be a self-complementary solution of $(\mathrm{E})$. By definition, this means that

$$
0=x_{\mathrm{E}}^{\mathrm{T}} \Pi_{\mathrm{H}} x_{\mathrm{E}}=2\left\|c_{\mathrm{E}}\right\|_{2}^{2} y_{0} .
$$

Consequently, $y_{0}=0$ and $x_{\mathrm{E}} \in \mathcal{A}_{\mathrm{H}} \cap \mathcal{K}_{\mathrm{H}}$. Moreover, $x_{\mathrm{E}} \neq 0$ due to the normalization constraint (11). 
5. Let $x_{\mathrm{E}} \in \mathcal{A}_{\mathrm{H}} \cap \mathcal{K}_{\mathrm{H}} \backslash\{0\}$. Since $\Pi_{\mathrm{H} \iota} \in$ int $\mathcal{K}_{\mathrm{H}}^{*}$, we obtain using (5) that

$$
\iota^{\mathrm{T}} \Pi_{\mathrm{H}} x_{\mathrm{E}}>0
$$

so that

$$
\frac{\iota^{\mathrm{T}} \Pi_{\mathrm{H}} \iota}{\iota^{\mathrm{T}} \Pi_{\mathrm{H}} x_{\mathrm{E}}} x_{\mathrm{E}} \in\left[b_{\mathrm{E}}+\left(\mathcal{A}_{\mathrm{H}} \cap \operatorname{Ker} b_{\mathrm{E}}^{\mathrm{T}}\right)\right] \cap \mathcal{K}_{\mathrm{H}}
$$

Remark from Theorem 2 and Theorem 3 that if (SD) is strongly feasible and $\left(x, x_{0}, z_{0}\right)$ is an optimal solution of (E), then $x / x_{0}$ is a self-complementary solution of (SD). Using the interior

point method [16], we can thus obtain an optimal solution to (SD) by solving the artificial program (E), for which we can choose an initial feasible solution $\iota \in \operatorname{int} \mathcal{K}_{\mathrm{SD}}$. We will see in the next section that even if (SD) is not strongly feasible (in which case it may not be solvable), it is still a good idea to solve the embedding (E), if the solution method generates a so-called weakly centered sequence.

\section{$5 \quad$ Weakly Centered Sequences}

Up to now, we did not use the special structure of the homogeneous program (H) in our study of the extended self-dual program (E). In this section however, we will focus on the full structure of (E), and we partition the decision variable as $x_{\mathrm{E}}=\left(x_{\mathrm{SD}}, x_{0}, z_{0}\right)$, just like we did in the homogeneous model previously. Similarly, we write

$$
\iota^{\mathrm{T}}=\left[\begin{array}{lll}
u_{\mathrm{SD}}^{\mathrm{T}}, & u_{0}, & v_{0}
\end{array}\right] .
$$

Throughout this section, we make the following assumption:

Assumption 1 The cone $\mathcal{K}_{S D}$ is solid, i.e. int $\mathcal{K}_{S D} \neq \emptyset$.

Since $\iota \in \operatorname{int} \mathcal{K}_{\mathrm{H}}=\left(\right.$ int $\left.\mathcal{K}_{\mathrm{SD}}\right) \times \Re_{++} \times \Re_{++}$, there holds

$$
u_{\mathrm{SD}} \in \operatorname{int} \mathcal{K}_{\mathrm{SD}}, \quad u_{0}>0, \quad v_{0}>0 .
$$

We can now formulate the model (E) as follows:

$$
\begin{array}{ll}
\min & \left(\iota^{\mathrm{T}} \Pi_{\mathrm{H}} \iota / 2\right) y_{0} \\
\text { s.t. } & x_{\mathrm{SD}}-y_{0} u_{\mathrm{SD}} \in\left(x_{0}-y_{0} u_{0}\right) b_{\mathrm{SD}}+\mathcal{A}_{\mathrm{SD}} \\
& z_{0}-y_{0} v_{0}=-c_{\mathrm{SD}}^{\mathrm{T}}\left(x_{\mathrm{SD}}-y_{0} u_{\mathrm{SD}}\right) \\
& \iota^{\mathrm{T}} \Pi_{\mathrm{H}} x_{\mathrm{E}}=\left(1+y_{0}\right) \iota^{\mathrm{T}} \Pi_{\mathrm{H}} \iota / 2 \\
& x_{\mathrm{SD}} \in \mathcal{K}_{\mathrm{SD}}, x_{0} \in \Re_{+}, z_{0} \in \Re_{+}, y_{0} \in \Re .
\end{array}
$$


Remark from (10) and Theorem 1 that

$$
\left(\iota^{\mathrm{T}} \Pi_{\mathrm{H}} \iota / 2\right) y_{0}=c_{\mathrm{E}}^{\mathrm{T}} x_{\mathrm{E}}=x_{0} z_{0}+\left(x^{\mathrm{T}} \Pi_{\mathrm{SD}} x\right) / 2 .
$$

In the sequel, we will analyze the behavior of weakly centered sequences for (E); the existence of such sequences will be demonstrated in Section 7 .

Definition 2 A sequence $x_{E}^{(k)}=\left(x_{S D}^{(k)}, x_{0}^{(k)}, z_{0}^{(k)}\right) \in\left(b_{E}+\mathcal{A}_{E}\right) \cap \mathcal{K}_{H}, k=1,2, \ldots$, is weakly centered if and only if there exists some constant $\omega \in(0,1)$ such that

$$
x_{0}^{(k)} z_{0}^{(k)} \geq \omega c_{E}^{T} x_{E}^{(k)}>0 \text { for all } k=1,2, \ldots,
$$

and $\lim _{k \rightarrow \infty} c_{E}^{T} x_{E}^{(k)}=0$.

Condition (16) is also known as the minimal centrality condition [25]. This condition holds true for all path-following algorithms, and for some potential reduction methods. In particular, Nesterov and Todd [18] developed a framework of primal-dual interior point algorithms for solving selfscaled conic convex programming, which is a subclass of conic convex programming that includes linear programming and semidefinite programming, among others. All their algorithms generate a sequence of weakly centered iterates. We remark here that if (SD) is a linear (semidefinite, selfscaled) programming problem, then (E) is also a linear (semidefinite, self-scaled) programming problem, since $\mathcal{K}_{\mathrm{H}}=\mathcal{K}_{\mathrm{SD}} \times \Re_{+} \times \Re_{+}$.

Since $c_{\mathrm{E}}^{\mathrm{T}} x_{\mathrm{E}}=\left(\iota^{\mathrm{T}} \Pi_{\mathrm{H}} \iota / 2\right) y_{0}$, see (9) and (10), it follows by definition that weakly centered sequences satisfy

$$
x_{0}^{(k)} z_{0}^{(k)} \geq \omega\left(\iota^{\mathrm{T}} \Pi_{\mathrm{H}} \iota / 2\right) y_{0}^{(k)}>0 \text { for all } k=1,2, \ldots
$$

This immediately implies the following result.

Lemma 3 Let $x_{E}^{(1)}, x_{E}^{(2)}, \ldots$ be a weakly centered sequence, then

$$
\lim _{k \rightarrow \infty} \frac{y_{0}^{(k)}}{x_{0}^{(k)}}=0 \Longleftrightarrow \lim _{k \rightarrow \infty} z_{0}^{(k)}=0,
$$

and

$$
\lim _{k \rightarrow \infty} \frac{y_{0}^{(k)}}{z_{0}^{(k)}}=0 \Longleftrightarrow \lim _{k \rightarrow \infty} x_{0}^{(k)}=0 .
$$

The lemma below shows a crucial property of weakly centered sequences: the components $x_{0}^{(k)}$ and $z_{0}^{(k)}$ avoid the boundary of the cone $\Re_{+}$essentially as much as possible. 
Lemma 4 Let $x_{E}=\left(x, x_{0}, z_{0}\right) \in\left(b_{E}+\mathcal{A}_{E}\right) \cap \mathcal{K}_{H}$ and $\omega \in(0,1)$ be such that $x_{0} z_{0} \geq \omega c_{E}^{T} x_{E}>0$. For any $x_{E}^{\prime}=\left(x^{\prime}, x_{0}^{\prime}, z_{0}^{\prime}\right) \in\left(b_{E}+\mathcal{A}_{E}\right) \cap \mathcal{K}_{H}$ there holds

$$
x_{0} \geq \frac{\omega}{1+\left(c_{E}^{T} x_{E}^{\prime} / c_{E}^{T} x_{E}\right)} x_{0}^{\prime}
$$

and

$$
z_{0} \geq \frac{\omega}{1+\left(c_{E}^{T} x_{E}^{\prime} / c_{E}^{T} x_{E}\right)} z_{0}^{\prime}
$$

Proof. Because $x_{\mathrm{E}}^{\prime}-x_{\mathrm{E}} \in \mathcal{A}_{\mathrm{E}}$ and $\Pi_{\mathrm{H}}\left(x_{\mathrm{E}}^{\prime}-x_{\mathrm{E}}\right) \in \mathcal{A}_{\mathrm{E}}^{\perp}$, there holds

$$
\begin{aligned}
0 & =\left(x_{\mathrm{E}}^{\prime}-x_{\mathrm{E}}\right)^{\mathrm{T}} \Pi_{\mathrm{H}}\left(x_{\mathrm{E}}^{\prime}-x_{\mathrm{E}}\right) \\
& =\left(x_{\mathrm{E}}^{\prime}\right)^{\mathrm{T}} \Pi_{\mathrm{H}} x_{\mathrm{E}}^{\prime}+x_{\mathrm{E}}^{\mathrm{T}} \Pi_{\mathrm{H}} x_{\mathrm{E}}-2 x_{\mathrm{E}}^{\mathrm{T}} \Pi_{\mathrm{H}} x_{\mathrm{E}}^{\prime} \\
& =2 c_{\mathrm{E}}^{\mathrm{T}}\left(x_{\mathrm{E}}^{\prime}+x_{\mathrm{E}}\right)-2 x^{\mathrm{T}} \Pi x^{\prime}-2\left(x_{0} z_{0}^{\prime}+z_{0}^{\prime} x_{0}\right) .
\end{aligned}
$$

Since $x \in \mathcal{K}$ and $\Pi x^{\prime} \in \mathcal{K}^{*}$, we have $x^{\mathrm{T}} \Pi x^{\prime} \geq 0$. We thus obtain from (17) that

$$
c_{\mathrm{E}}^{\mathrm{T}}\left(x_{\mathrm{E}}^{\prime}+x_{\mathrm{E}}\right) \geq x_{0} z_{0}^{\prime}+z_{0} x_{0}^{\prime}
$$

The lemma follows by multiplying (18) with $x_{0} / c_{\mathrm{E}}^{\mathrm{T}}\left(x_{\mathrm{E}}^{\prime}+x_{\mathrm{E}}\right)$ and $z_{0} / c_{\mathrm{E}}^{\mathrm{T}}\left(x_{\mathrm{E}}^{\prime}+x_{\mathrm{E}}\right)$ respectively.

The argumentation that is used in the proof of Lemma 4 is due to Güler and Ye [5].

Theorem 4 below shows why weakly centered sequences are so interesting in the context of self-dual embeddings. Namely, if we can generate a weakly centered sequence for (E) then we can also solve (SD), whenever it has a complementary solution or an improving direction. In other cases, (SD) must be either weakly feasible or weakly infeasible, and we can generate a sequence of solutions for (SD), for which the amount of constraint violation converges to zero and the corresponding objective values are in the limit contained in the interval $\left[p_{\mathrm{SD}}^{-}, p_{\mathrm{SD}}^{*}\right]$.

Theorem 4 Let $x_{E}^{(k)}=\left(x^{(k)}, x_{0}^{(k)}, z_{0}^{(k)}\right), k=1,2, \ldots$, be a weakly centered sequence for $(E)$. There holds

1. $\liminf _{k \rightarrow \infty} x_{0}^{(k)}>0$ if and only if (SD) has a self-complementary solution. Moreover, if (SD) has a self-complementary solution then $x_{S D}^{(k)} / x_{0}^{(k)}, k=1,2, \ldots$ is a bounded sequence and therefore it has a cluster point $x_{S D}^{(\infty)}$. Any such cluster point $x_{S D}^{(\infty)}$ is a self-complementary solution of $(S D)$.

2. $\liminf \operatorname{in}_{k \rightarrow \infty} z_{0}^{(k)}>0$ if and only if (SD) is strongly infeasible. Moreover, if (SD) is strongly infeasible, then $x_{S D}^{(k)} / z_{0}^{(k)}, k=1,2, \ldots$ is a bounded sequence and therefore it has a cluster point $x_{S D}^{(\infty)}$. Any such cluster point $x_{S D}^{(\infty)}$ is an improving direction of $(S D)$. 
3. If $\lim _{k \rightarrow \infty} z_{0}^{(k)}=0$, then $x_{S D}^{(k)} / x_{0}^{(k)}$ is a sequence in $\mathcal{K}_{S D}$ for which

$$
\lim _{k \rightarrow \infty} \operatorname{dist}\left(\frac{x_{S D}^{(k)}}{x_{0}^{(k)}}, b_{S D}+\mathcal{A}_{S D}\right)=0, \quad \lim _{k \rightarrow \infty} \frac{c_{S D}^{T} x_{S D}^{(k)}+z_{0}^{(k)}}{x_{0}^{(k)}}=0 \leq p_{S D}^{*}
$$

4. If $\lim _{k \rightarrow \infty} z_{0}^{(k)} / x_{0}^{(k)}=\infty$, then (SD) is infeasible, and $x_{S D}^{(k)} / z_{0}^{(k)}$ is an improving direction sequence in $\mathcal{K}_{S D}$, viz.

$$
\lim _{k \rightarrow \infty} \operatorname{dist}\left(\frac{x_{S D}^{(k)}}{z_{0}^{(k)}}, \mathcal{A}_{S D}\right)=0, \quad \lim _{k \rightarrow \infty} \frac{c_{S D}^{T} x_{S D}^{(k)}}{z_{0}^{(k)}}=-1 .
$$

\section{Proof.}

1. Observe from Theorem 3 and the discussion in Section 4.1 we can conclude that (SD) has a self-complementary solution if and only if (E) has an optimal solution $\left(x_{\mathrm{SD}}^{*}, x_{0}^{*}, 0\right)$ with $x_{0}^{*}>0$. From Lemma 4 , we know that if (E) has an optimal solution $\left(x_{\mathrm{SD}}^{*}, x_{0}^{*}, 0\right)$ with $x_{0}^{*}>0$, then

$$
\liminf _{k \rightarrow \infty} x_{0}^{(k)} \geq \omega x_{0}^{*}>0 .
$$

The converse is also true, because the sequence $\left(x_{\mathrm{SD}}^{(k)}, x_{0}^{(k)}, z_{0}^{(k)}\right)$ is bounded (and hence it has a cluster point, which must be an optimal solution to (E)).

2. Similarly, (SD) is strongly infeasible if and only if the embedding (E) has an optimal solution $\left(x_{\mathrm{SD}}^{*}, 0, z_{0}^{*}\right)$ with $z_{0}^{*}>0$, which is equivalent with the relation

$$
\liminf _{k \rightarrow \infty} z_{0}^{(k)} \geq \omega z_{0}^{*}>0
$$

3. Suppose that $\lim _{k \rightarrow \infty} z_{0}^{(k)}=0$, so that, using Lemma 3 ,

$$
\lim _{k \rightarrow \infty} \frac{y_{0}^{(k)}}{x_{0}^{(k)}}=0 .
$$

Combining this with (12)-(13), we obtain

$$
\lim _{k \rightarrow \infty} \operatorname{dist}\left(\frac{x_{\mathrm{SD}}^{(k)}}{x_{0}^{(k)}}, b_{\mathrm{SD}}+\mathcal{A}_{\mathrm{SD}}\right)=0, \quad \lim _{k \rightarrow \infty} \frac{c_{\mathrm{SD}}^{\mathrm{T}} x_{\mathrm{SD}}^{(k)}+z_{0}^{(k)}}{x_{0}^{(k)}}=0 .
$$

4. Suppose that $\lim _{k \rightarrow \infty} z_{0}^{(k)} / x_{0}^{(k)}=\infty$. Then $x_{0}^{(k)} \rightarrow 0$ so that using Lemma 3 ,

$$
\lim _{k \rightarrow \infty} \frac{y_{0}^{(k)}}{z_{0}^{(k)}}=0
$$


Combining this with (12)-(13), we obtain

$$
\lim _{k \rightarrow \infty} \operatorname{dist}\left(\frac{x_{\mathrm{SD}}^{(k)}}{z_{0}^{(k)}}, \mathcal{A}_{\mathrm{SD}}\right)=0, \quad \lim _{k \rightarrow \infty} \frac{c_{\mathrm{SD}}^{\mathrm{T}} x_{\mathrm{SD}}^{(k)}}{z_{0}^{(k)}}=-1 .
$$

By definition, $x_{\mathrm{SD}}^{(k)} / z_{0}^{(k)}$ is then an improving direction sequence, which implies that (SD) is infeasible (see the discussion on Farkas-type duality in Section 2).

Theorem 5 Let $x_{E}^{(k)}=\left(x^{(k)}, x_{0}^{(k)}, z_{0}^{(k)}\right), k=1,2, \ldots$, be a weakly centered sequence for $(E)$. There holds

$$
p_{S D}^{*} \geq \limsup _{k \rightarrow \infty} \frac{z_{0}^{(k)}}{x_{0}^{(k)}} .
$$

Proof. It is known from Theorem 4 that $\liminf _{k \rightarrow \infty} z_{0}^{(k)}>0$ if (SD) is strongly infeasible. Using e.g. Lemma 3 , it follows that $\liminf _{k \rightarrow \infty} x_{0}^{(k)}=0$, and hence

$$
p_{\mathrm{SD}}^{*}=\lim _{k \rightarrow \infty} \frac{z_{0}^{(k)}}{x_{0}^{(k)}}=\infty .
$$

Now suppose that (SD) is not strongly infeasible, or equivalently, $\liminf _{k \rightarrow \infty} z_{0}^{(k)}=0$. For this case, we know from Theorem 4 that $x_{\mathrm{SD}}^{(k)} / x_{0}^{(k)}$ is a sequence in $\mathcal{K}_{\mathrm{SD}}$, with

$$
\lim _{k \rightarrow \infty} \operatorname{dist}\left(\frac{x_{\mathrm{SD}}^{(k)}}{x_{0}^{(k)}}, b_{\mathrm{SD}}+\mathcal{A}\right)=0
$$

Therefore, we have the following inequality for the subvalue $p_{\mathrm{SD}}^{-}$of $(\mathrm{SD})$ :

$$
p_{\mathrm{SD}}^{-} \leq \liminf _{k \rightarrow \infty} \frac{c_{\mathrm{SD}}^{\mathrm{T}} x_{\mathrm{SD}}^{(k)}}{x_{0}^{(k)}}=-\limsup _{k \rightarrow \infty} \frac{z_{0}^{(k)}}{x_{0}^{(k)}} .
$$

Using (6), the theorem follows.

Remark 4 It follows from Theorem 5 that if $\lim \sup _{k \rightarrow \infty}\left(z_{0}^{(k)} / x_{0}^{(k)}\right)=\infty$, then (SD) is infeasible.

Remark 5 Theorem 5 also shows that if there is no duality gap, i.e. $p_{S D}^{*}=0$, then

$$
\lim _{k \rightarrow \infty}\left(z_{0}^{(k)} / x_{0}^{(k)}\right)=0 .
$$




\section{The Primal-Dual Model}

Up to now, we have only considered self-dual embeddings for self-dual programs. However, the self-dual embedding technique is applicable to general closed conic convex programs, simply by combining the original primal and dual programs into a single, self-dual program. To be more specific, consider a closed conic convex program $\operatorname{CP}(b, c, \mathcal{A}, \mathcal{K})$, and let

$$
b_{\mathrm{SD}}:=\left[\begin{array}{ll}
b^{\mathrm{T}}, & c^{\mathrm{T}}
\end{array}\right]^{\mathrm{T}}, \quad c_{\mathrm{SD}}:=\left[\begin{array}{ll}
c^{\mathrm{T}}, & b^{\mathrm{T}}
\end{array}\right]^{\mathrm{T}},
$$

and

$$
\mathcal{A}_{\mathrm{SD}}:=\mathcal{A} \times \mathcal{A}^{\perp}, \quad \mathcal{K}_{\mathrm{SD}}:=\mathcal{K} \times \mathcal{K}^{*}
$$

The program (SD) is easily seen to be $\Pi_{\mathrm{SD}}$ self-dual, with

$$
\Pi_{\mathrm{SD}}=\left[\begin{array}{cc}
0 & I \\
I & 0
\end{array}\right] .
$$

If $\operatorname{CP}(b, c, \mathcal{A}, \mathcal{K})$ is both primal and dual feasible, then $(b, c, \mathcal{A}, \mathcal{K})$ and (SD) are equivalent, as follows from the weak duality relation for conic convex programming. Therefore, it is interesting to study the self-dual embedding (E) of the above constructed self-dual model (SD). Since our basic interest lies in the connection with the original program $\mathrm{CP}(b, c, \mathcal{A}, \mathcal{K})$, we partition $x_{\mathrm{SD}}$ and $u_{\mathrm{SD}}$ as follows:

$$
x_{\mathrm{SD}}^{\mathrm{T}}=\left[x^{\mathrm{T}}, z^{\mathrm{T}}\right], \quad u_{\mathrm{SD}}^{\mathrm{T}}=\left[\begin{array}{ll}
u_{p}^{\mathrm{T}}, & u_{d}^{\mathrm{T}}
\end{array}\right] .
$$

Since (SD) is self-dual, the results of Theorems 4 and 5 are applicable. However, if (SD) has no self-complementary solution, it is not fully equivalent with the original conic convex program $\operatorname{CP}(b, c, \mathcal{A}, \mathcal{K})$. Below, we will therefore use the special structure of the primal-dual model, to deduce as much information as possible for $\operatorname{CP}(b, c, \mathcal{A}, \mathcal{K})$ and its dual.

Theorem 6 Consider a closed conic convex program $C P(b, c, \mathcal{A}, \mathcal{K})$ and let $x_{E}^{(k)}, k=1,2, \ldots$, be a weakly centered sequence for the self-dual embedding $(E)$, where

$$
C P\left(b_{S D}, c_{S D}, \mathcal{A}_{S D}, \mathcal{K}_{S D}\right)
$$

is defined as in (19)-(20). Then

1. (SD) has a self-complementary solution if and only if $\lim _{\sup _{k \rightarrow \infty}} x_{0}^{(k)}>0$.

2. (SD) is strongly infeasible if and only if $\lim \sup _{k \rightarrow \infty} z_{0}^{(k)}>0$. 
3. If $\lim _{k \rightarrow \infty} z_{0}^{(k)}=0$ then

$$
\lim _{k \rightarrow \infty} \operatorname{dist}\left(\frac{x^{(k)}}{x_{0}^{(k)}}, b+\mathcal{A}\right)=0, \quad \limsup _{k \rightarrow \infty} \frac{c^{T} x^{(k)}+z_{0}^{(k)}}{x_{0}^{(k)}} \leq p^{*}
$$

and

$$
d^{*} \geq-\liminf _{k \rightarrow \infty} \frac{c^{T} x^{(k)}}{x_{0}^{(k)}} \geq-\limsup _{k \rightarrow \infty} \frac{c^{T} x^{(k)}+z_{0}^{(k)}}{x_{0}^{(k)}}
$$

Proof. The cases of self-complementarity and strong infeasibility are known from Theorem 4.

If $\lim _{k \rightarrow \infty} z_{0}^{(k)}=0$ then neither (P) nor (D) is strongly infeasible, and it follows from (1) that

$$
p^{*}=-d^{-}, \quad p^{-}=-d^{*} .
$$

Moreover, we know from Theorem 4 that

$$
\lim _{k \rightarrow \infty} \operatorname{dist}\left(\frac{x^{(k)}}{x_{0}^{(k)}}, b+\mathcal{A}\right)=0
$$

so that by definition of the subvalue,

$$
p^{-} \leq \liminf _{k \rightarrow \infty} \frac{c^{\mathrm{T}} x^{(k)}}{x_{0}^{(k)}}, \quad d^{-} \leq \liminf _{k \rightarrow \infty} \frac{b^{\mathrm{T}} z^{(k)}}{x_{0}^{(k)}} .
$$

Moreover, using (13) and Lemma 3, it follows that

$$
\lim _{k \rightarrow \infty} \frac{c^{\mathrm{T}} x^{(k)}+b^{\mathrm{T}} z^{(k)}+z_{0}^{(k)}}{x_{0}^{(k)}}=0 .
$$

Combining (21)-(23), the theorem follows.

Some remarks concerning Theorem 6 have to be made:

Remark 6 The case of self-complementarity was already known from Theorem 4, which also states that self-complementarity will be demonstrated by a self-complementary solution, say $x_{S D}^{*}=$ $\left(x^{*}, z^{*}\right)$. It is obvious that $\left(x^{*}, z^{*}\right)$ is then a complementary solution pair for $(P)$ and $(D)$.

Remark 7 Similarly, we know from Theorem 4 that strong infeasibility will be demonstrated by an improving direction, say $x_{S D}^{*}=\left(x^{*}, z^{*}\right)$. In this case, we have $c^{T} x^{*}+b^{T} z^{*}<0$, so that either $c^{T} x^{*}$ and $b^{T} z^{*}$ are both negative, or exactly one of the quantities $c^{T} x^{*}$ and $b^{T} z^{*}$ is negative, say $c^{T} x^{*}<0$ and $b^{T} z^{*} \geq 0$. In the former case, $z^{*}$ and $x^{*}$ demonstrate primal and dual strong infeasibility respectively. In the latter case, it follows that $(D)$ is strongly infeasible, but we do not have complete information about $(P):(P)$ can be either unbounded or infeasible. 
Remark that by definition, (P) is unbounded if and only if $p^{*}=-\infty$, and (D) is infeasible if and only if $d^{*}=\infty$. The following is therefore a consequence of Theorem 6 .

Corollary 1 If $(P)$ is unbounded and $(D)$ is weakly infeasible, then

$$
\lim _{k \rightarrow \infty} z_{0}^{(k)}=0, \quad \lim _{k \rightarrow \infty} \frac{c^{T} x^{(k)}+z_{0}^{(k)}}{x_{0}^{(k)}}=-\infty .
$$

Conversely, if

$$
\lim _{k \rightarrow \infty} z_{0}^{(k)}=0, \quad \lim _{k \rightarrow \infty} \frac{c^{T} x^{(k)}+z_{0}^{(k)}}{x_{0}^{(k)}}=-\infty,
$$

then $(D)$ is weakly infeasible and either $(P)$ is unbounded or $p^{*}>-d^{*}$.

Remark 8 If $(P)$ is strongly feasible, then $p^{*}=-d^{*}$, see (2), and Corollary 1 characterizes the case of primal unboundedness. In general however, we cannot conclude unboundedness from (24), as is illustrated later in this chapter by Example $\%$.

There are still some cases that are not described by Theorem 6 and Corollary 1 . Namely, it can happen that

- $p^{*}$ is finite, and $p^{*}=-d^{*}$, but $(\mathrm{P})$ is not solvable,

- $p^{*}$ and $d^{*}$ are finite, but $p^{*}+d^{*}>0$,

- $(\mathrm{P})$ is weakly feasible, but (D) is weakly infeasible,

- $(\mathrm{P})$ and (D) are both weakly infeasible.

In all these remaining cases, we will obtain some partial information, based on the value of $\lim \sup _{k \rightarrow \infty} z_{0}^{(k)} / x_{0}^{(k)}$.

Theorem 7 Consider a closed conic convex program $C P(b, c, \mathcal{A}, \mathcal{K})$ and let $x_{E}^{(k)}, k=1,2, \ldots$, be a weakly centered sequence for the self-dual embedding $(E)$, where we use the primal-dual model $C P\left(b_{S D}, c_{S D}, \mathcal{A}_{S D}, \mathcal{K}_{S D}\right)$ as defined in (19)-(20). Suppose that $\lim _{k \rightarrow \infty} x_{0}^{(k)}=\lim _{k \rightarrow \infty} z_{0}^{(k)}=0$ and

$$
\liminf _{k \rightarrow \infty} \frac{c^{T} x^{(k)}+z_{0}^{(k)}}{x_{0}^{(k)}}>-\infty, \quad \liminf _{k \rightarrow \infty} \frac{b^{T} z^{(k)}+z_{0}^{(k)}}{x_{0}^{(k)}}>-\infty .
$$

Then

1. If $\lim \sup _{k \rightarrow \infty} z_{0}^{(k)} / x_{0}^{(k)}=\infty$, then (SD) is weakly infeasible, and $p^{*} \neq-d^{*}$. Hence, $p^{*}+d^{*}=$ $\infty$. Moreover, $x_{S D}^{(k)} / z_{0}^{(k)}, k=1,2, \ldots$, is an improving direction sequence. 
2. If $0<\lim _{\sup _{k \rightarrow \infty}} z_{0}^{(k)} / x_{0}^{(k)}<\infty$, then $p^{*} \neq-d^{*}$ and neither $(P)$ nor $(D)$ is strongly feasible. Moreover, $\left\|x^{(k)}\right\|>0$ for all sufficiently large $k$, and any cluster point of the sequence $x^{(k)} /\left\|x^{(k)}\right\|$ is a nonzero lower level direction, demonstrating the fact that $(D)$ is not strongly feasible.

3. If $\lim \sup _{k \rightarrow \infty} z_{0}^{(k)} / x_{0}^{(k)}=0$, we have the following:

- If $\lim _{k \rightarrow \infty}\left\|x^{(k)}\right\| / x_{0}^{(k)}=\infty$, then (D) is not strongly feasible. Moreover, $\left\|x^{(k)}\right\|$ is positive for all sufficiently large $k$, and any cluster point of the sequence $x^{(k)} /\left\|x^{(k)}\right\|$ is a nonzero lower level direction, demonstrating the fact that $(D)$ is not strongly feasible.

- Otherwise, i.e. if $\liminf _{k \rightarrow \infty}\left\|x^{(k)}\right\| / x_{0}^{(k)}<\infty$, then $(P)$ is solvable and weakly feasible. Moreover, any cluster point of the sequence $x^{(k)} / x_{0}^{(k)}$ is an optimal solution for $(P)$ and $\liminf _{k \rightarrow \infty} c^{T} z^{(k)} / x_{0}^{(k)}=-p^{*}$.

\section{Proof.}

1. It is already known from Theorem 4 that if $\lim _{\sup _{k \rightarrow \infty}} z_{0}^{(k)} / x_{0}^{(k)}=\infty$, then (SD) is weakly infeasible, and $x_{\mathrm{SD}}^{(k)} / z_{0}^{(k)}, k=1,2, \ldots$, is an improving direction sequence. Using Corollary 1, we have $p^{*}>-\infty$ and $d^{*}>-\infty$, and it follows that $p^{*}+d^{*}=\infty$ and $p^{*} \neq-d^{*}$.

2. We use that $p^{*}>-\infty$ and $d^{*}>-\infty$ to conclude from Theorem 5 that if

$$
\limsup _{k \rightarrow \infty} z_{0}^{(k)} / x_{0}^{(k)}>0
$$

then $p^{*}+d^{*}>0$. Together with (2), this implies that neither (P) nor (D) is strongly feasible. Using Theorem 6 , we know that the sequence $x^{(k)} / x_{0}^{(k)}$ cannot have any cluster point. Namely, if $x$ is such a cluster point then $x \in(b+\mathcal{A}) \cap \mathcal{K}$ and $c^{\mathrm{T}} x<p^{*}$, a contradiction. Consequently, $\lim _{k \rightarrow \infty}\left\|x^{(k)}\right\| / x_{0}=\infty$ and we obtain from (12) that any cluster point $x^{*}$ of the sequence $x^{(k)} /\left\|x^{(k)}\right\|$ is a nonzero direction, i.e. $0 \neq x^{*} \in \mathcal{A} \cap \mathcal{K}$. Dividing (13) by $x_{0}$, and using Lemma 3, we have

$$
\limsup _{k \rightarrow \infty} \frac{c^{\mathrm{T}} x^{(k)}}{x_{0}^{(k)}}=-\liminf _{k \rightarrow \infty} \frac{b^{\mathrm{T}} z^{(k)}+z_{0}^{(k)}}{x_{0}^{(k)}}<\infty,
$$

where the inequality is an assumption of the lemma. Since $x_{0}^{(k)}=o\left(\left\|x^{(k)}\right\|\right)$, this inequality implies that $c^{\mathrm{T}} x^{*} \leq 0$, i.e. $x^{*}$ is a lower-level direction.

3. Suppose that $\lim \sup _{k \rightarrow \infty} z_{0}^{(k)} / x_{0}^{(k)}=0$. The case that $\lim _{k \rightarrow \infty}\left\|x^{(k)}\right\| / x_{0}^{(k)}=\infty$ is completely analogous to the case $0<\lim \sup _{k \rightarrow \infty} z_{0}^{(k)} / x_{0}^{(k)}<\infty$, which has been treated above. 
If $\liminf _{k \rightarrow \infty}\left\|x^{(k)}\right\| / x_{0}^{(k)}<\infty$, then the sequence $x^{(k)} / x_{0}^{(k)}$ must have a cluster point, and it follows from Theorem 6 that such a cluster point is an optimal solution for (P). We also know from Theorem 6 that there is no complementary solution pair, and hence $\lim _{k \rightarrow \infty}\left\|z^{(k)}\right\| / x_{0}^{(k)}=\infty$. We have already seen above that this implies that any cluster point of $z^{(k)} /\left\|z^{(k)}\right\|$ is a dual lower level direction, demonstrating weak feasibility of (P). From (13) and $\lim \sup _{k \rightarrow \infty} z_{0}^{(k)} / x_{0}^{(k)}=0$, it follows that $\liminf _{k \rightarrow \infty} c^{\mathrm{T}} z^{(k)} / x_{0}^{(k)}=-p^{*}$.

Solving the self-dual embedding (E) is really equivalent to solving $(\mathrm{P})$ if $(\mathrm{P})$ is strongly feasible, as the following theorem shows.

Theorem 8 Consider a closed conic convex program $C P(b, c, \mathcal{A}, \mathcal{K})$, and suppose that it is primal strongly feasible. Let $x_{E}^{(k)}, k=1,2, \ldots$, be a weakly centered sequence for the self-dual embedding $(E)$, where $C P\left(b_{S D}, c_{S D}, \mathcal{A}_{S D}, \mathcal{K}_{S D}\right)$ is defined as in (19)-(20). Then $C P(b, c, \mathcal{A}, \mathcal{K})$ is

1. Solvable if and only if

$$
\liminf _{k \rightarrow \infty} x_{0}^{(k)}>0
$$

Moreover, if $(P)$ is solvable, then any cluster point of the (bounded) sequence

$$
\left\{\left(\frac{x^{(k)}}{x_{0}^{(k)}}, \frac{z^{(k)}}{x_{0}^{(k)}}\right) \mid k=1,2, \ldots\right\},
$$

is a complementary solution pair. (Cf. Theorem 6.)

2. Unbounded and dual strongly infeasible if and only if

$$
\liminf _{k \rightarrow \infty} z_{0}^{(k)}>0
$$

Moreover, if (D) is strongly infeasible then any cluster point of the (bounded) sequence $x^{(1)}, x^{(2)}, \ldots$ is a primal improving direction, certifying the dual strong infeasibility. (Cf. Theorem 6 and Remark \%.)

3. Unbounded and dual weakly infeasible if and only if

$$
\lim _{k \rightarrow \infty} z_{0}^{(k)}=0, \quad \lim _{k \rightarrow \infty} \frac{c^{T} x^{(k)}+z_{0}^{(k)}}{x_{0}^{(k)}}=-\infty .
$$

Moreover, if $(D)$ is weakly infeasible then $x^{(k)} / z_{0}^{(k)}, k=1,2, \ldots$ is a primal improving direction sequence, certifying the dual weak infeasibility. (Cf. Corollary 1.) 
4. Dual weakly feasible and not primal solvable, if and only if

$$
\lim _{k \rightarrow \infty} x_{0}^{(k)}=0, \quad \lim _{k \rightarrow \infty} z_{0}^{(k)} / x_{0}^{(k)}=0 .
$$

Moreover, if $(D)$ is feasible and $(P)$ is not solvable, then

- $z^{(k)} / x_{0}^{(k)}, k=1,2, \ldots$, is a bounded sequence, and any cluster point of this sequence is a dual optimal solution.

- Any cluster point of the sequence $x^{(k)}, k=1,2, \ldots$ is a nonzero lower level direction, certifying that $(D)$ is not strongly feasible.

- $x^{(k)} / x_{0}^{(k)}, k=1,2, \ldots$ is a sequence of approximate primal solutions, with

$$
\lim _{k \rightarrow \infty} \frac{c^{T} x^{(k)}}{x_{0}^{(k)}}=p^{*}, \quad \lim _{k \rightarrow \infty} \operatorname{dist}\left(\frac{x^{(k)}}{x_{0}^{(k)}}, b+\mathcal{A}\right)=0 .
$$

(Cf. Theorem 7.)

Applying Theorem 6, Corollary 1 and Theorem 7, it is straightforward to prove Theorem 8.

Only slightly weaker results than those of Theorem 8 hold under the condition that $p^{*}=-d^{*}$, without requiring primal strong feasibility. Such results are then applicable to Ramana's regularized semidefinite programs [21]. See De Klerk, Roos and Terlaky [7] for a discussion of the self-dual embedding for regularized semidefinite programs.

\subsection{Examples in Semidefinite Programming}

Several primal-dual interior point algorithms have been extended from linear to semidefinite programming, see [8, 9, 15, 17, 24, 23], among others. All these algorithms generate a sequence of weakly centered iterates, so that all results of Section 6 are applicable.

We will illustrate the theory of weakly centered sequences for (E) with some semidefinite programming problems, i.e. $\mathcal{K}=\mathcal{K}^{*}=\mathcal{H}_{+}$. We continue with our convention that given a Hermitian matrix $Y \in \mathcal{H}^{(n)}$, the lower case symbol $y$ denotes $\operatorname{vec}_{\mathrm{H}} Y$, which is the coordinate vector of $Y$ with respect to a fixed orthonormal basis of the real linear space $\mathcal{H}^{(n)}$ of Hermitian $\bar{n} \times \bar{n}$ matrices. Letting $n$ denote the dimension of $\mathcal{H}^{(n)}$, i.e. $n=\bar{n}^{2}$, it follows that $y \in \Re^{n}$. The pair of primal and dual semidefinite programming problems is

$$
\text { (P) } \inf \left\{C \bullet X \mid X \in(B+\mathcal{A}) \cap \mathcal{H}_{+}\right\},
$$

and

$$
\text { (D) } \inf \left\{B \bullet Z \mid Z \in\left(C+\mathcal{A}^{\perp}\right) \cap \mathcal{H}_{+}\right\} \text {. }
$$




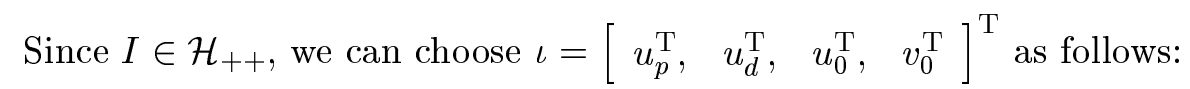

$$
U_{p}=U_{d}=I, \quad u_{0}=v_{0}=1 \text {. }
$$

With this choice, there holds

$$
\frac{\iota^{\mathrm{T}} \Pi_{\mathrm{H}} \iota}{2}=\bar{n}+1
$$

We obtain the following formulation of the extended self-dual model (E) from (12)-(14), by specializing it to semidefinite programming.

$$
\begin{array}{ll}
\min & (\bar{n}+1) y_{0} \\
\text { s.t. } & X-y_{0} I \in\left(x_{0}-y_{0}\right) B+\mathcal{A} \\
& Z-y_{0} I \in\left(x_{0}-y_{0}\right) C+\mathcal{A}^{\perp} \\
& z_{0}-y_{0}=-C \bullet\left(X-y_{0} I\right)-B \bullet\left(Z-y_{0} I\right) \\
& \operatorname{tr} X+\operatorname{tr} Z+x_{0}+z_{0}=\left(1+y_{0}\right)(\bar{n}+1) \\
& X \succeq 0, Z \succeq 0, x_{0} \geq 0 . z_{0} \geq 0, y_{0} \in \Re .
\end{array}
$$

Weakly centered sequences will now be parameterized by a continuous parameter $\epsilon>0$ such that

$$
\lim _{\epsilon \downarrow 0} y_{0}(\epsilon)=0 .
$$

We will only discuss those difficult cases where $\lim _{\epsilon \downarrow 0}\left(x_{0}(\epsilon)+z_{0}(\epsilon)\right)=0$.

First, we consider a weakly infeasible problem.

Example 1 (Weakly infeasible) Let $\bar{n}=2$ and

$$
B=\left[\begin{array}{ll}
0 & 1 \\
& 0
\end{array}\right], \quad C=\left[\begin{array}{ll}
0 & 0 \\
& 1
\end{array}\right], \quad \mathcal{A}=\left\{X \mid X=\left[\begin{array}{cc}
0 & 0 \\
& x_{22}
\end{array}\right]\right\} .
$$

The primal is weakly infeasible,

$$
p^{*}=\inf \left\{x_{22} \mid X=\left[\begin{array}{cc}
0 & 1 \\
& x_{22}
\end{array}\right] \succeq 0\right\}=\infty
$$

and the dual is strongly feasible and unbounded,

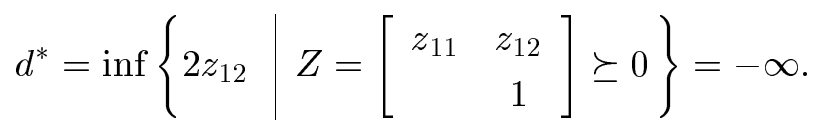

We construct a weakly centered sequence for $0<\epsilon \leq 1 / 3$ as follows:

$$
X(\epsilon)=\epsilon^{3} I+\left[\begin{array}{cc}
0 & \epsilon^{2} \\
& \epsilon
\end{array}\right], \quad Z(\epsilon)=\epsilon^{3} I+\left[\begin{array}{cc}
3-\left(2 \epsilon+2 \epsilon^{2}+3 \epsilon^{3}\right) & -\epsilon \\
& \epsilon^{2}
\end{array}\right],
$$




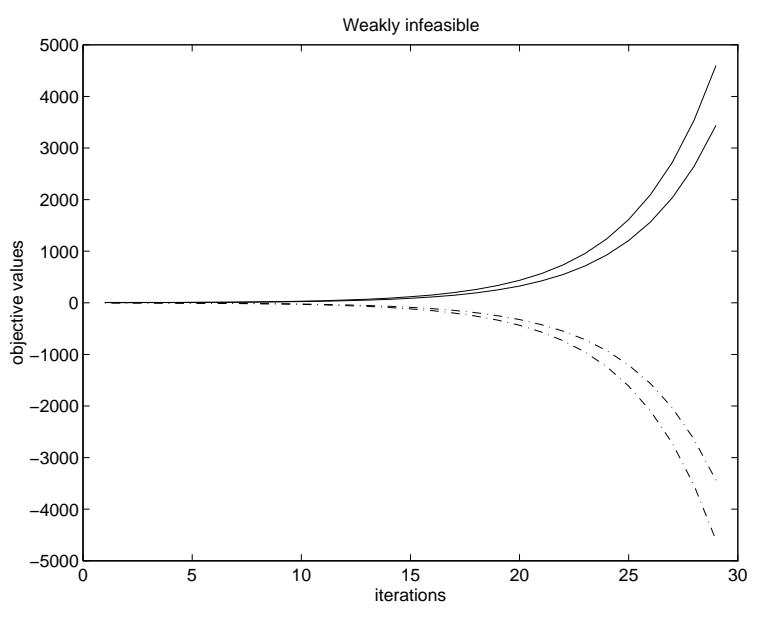

Figure 1: Example 1

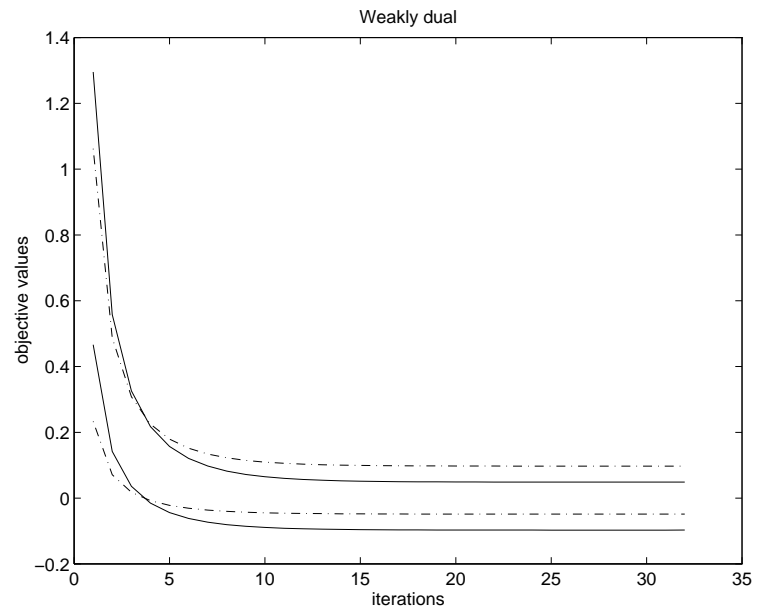

Figure 2: Example 2

$$
y_{0}(\epsilon)=\epsilon^{3}, \quad x_{0}(\epsilon)=\epsilon^{2}+\epsilon^{3}, \quad z_{0}(\epsilon)=\epsilon+\epsilon^{3} .
$$

We see that

$$
\lim _{\epsilon \downarrow 0} z_{0}(\epsilon)=0, \quad \lim _{\epsilon \downarrow 0} \frac{B \bullet Z(\epsilon)+z_{0}(\epsilon)}{x_{0}(\epsilon)}=-\infty,
$$

which indeed implies that the dual is unbounded and the primal is weakly infeasible, see Theorem 8. Finally, notice that

$$
\lim _{\epsilon \downarrow 0} \frac{z_{0}(\epsilon)}{x_{0}(\epsilon)}=\infty
$$

In order to be able to solve the self-dual embedding $(\mathrm{E})$, we specialized the predictor-corrector algorithm for semidefinite programming (with Nesterov-Todd type primal-dual directions [18, 17, 24]) to the special structure of (E). The plots below show the numerical results for the examples in this section. The solid lines represent the primal objective values

$$
\frac{C \bullet X^{(k)}}{x_{0}^{(k)}-y_{0}^{(k)}}, \quad \frac{C \bullet X^{(k)}+z_{0}^{(k)}}{x_{0}^{(k)}-y_{0}^{(k)}},
$$

whereas the dual objective values

$$
\frac{B \bullet Z^{(k)}}{x_{0}^{(k)}-y_{0}^{(k)}}, \quad \frac{B \bullet Z^{(k)}+z_{0}^{(k)}}{x_{0}^{(k)}-y_{0}^{(k)}}
$$

are represented by dashed lines. Recall from Theorem 6 that in the limit, $\left(C \bullet X^{(k)}+z_{0}^{(k)}\right) /\left(x_{0}^{(k)}-y_{0}^{(k)}\right)$ and $\left(B \bullet Z^{(k)}+z_{0}^{(k)}\right) /\left(x_{0}^{(k)}-y_{0}^{(k)}\right)$ provide lower bounds on $p^{*}$ and $d^{*}$ respectively.

The next example, which is from Vandenberghe and Boyd [27], gives a feasible problem, where strong duality fails to hold. 
Example 2 (Weakly dual) Let $\bar{n}=3$ and

$$
\begin{gathered}
B=\left[\begin{array}{ccc}
0 & 1 / 3 & 0 \\
& 0 & 0 \\
& 1 / 3
\end{array}\right], \quad C=\left[\begin{array}{ccc}
0 & -1 / 3 & 0 \\
& 0 & 0 \\
& & 2 / 3
\end{array}\right], \\
\mathcal{A}=\left\{X \mid X=\left[\begin{array}{ccc}
x_{11} & -x_{33} / 2 & x_{13} \\
& 0 & x_{23} \\
& & x_{33}
\end{array}\right]\right\},
\end{gathered}
$$

so that the primal is solvable and weakly feasible,

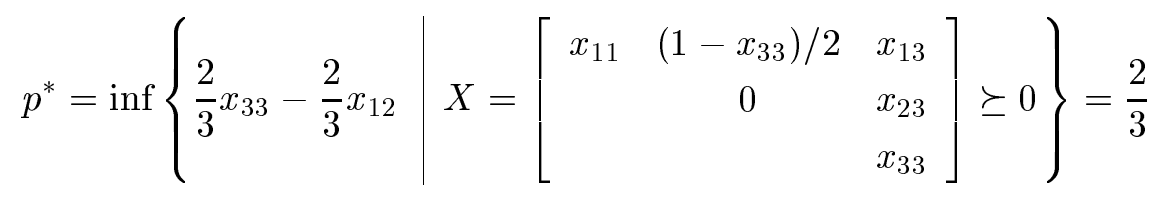

and the dual is also solvable and weakly feasible,

$$
d^{*}=\inf \left\{\frac{1}{3} z_{33}+\frac{2}{3} z_{12} \mid Z=\left[\begin{array}{ccc}
0 & z_{33}-1 & 0 \\
& z_{22} & 0 \\
& & z_{33}
\end{array}\right] \succeq 0\right\}=\frac{1}{3} .
$$

Remark that $p^{*}+d^{*}=1>0$ so that strong duality fails. A weakly centered sequence for $0<\epsilon \leq 1 / 2$ is given by

$$
\begin{gathered}
X(\epsilon)=\epsilon^{2} I+\left[\begin{array}{ccc}
1 & \epsilon / 2 & 0 \\
& 0 & 0 \\
& & 0
\end{array}\right], \quad Z(\epsilon)=\epsilon^{2} I+\left[\begin{array}{ccc}
0 & -\epsilon & 0 \\
& 3-\left(2 \epsilon+4 \epsilon^{2}\right) & 0 \\
& & 0
\end{array}\right], \\
y_{0}(\epsilon)=\epsilon^{2}, \quad x_{0}(\epsilon)=\epsilon+\epsilon^{2}, \quad z_{0}(\epsilon)=\epsilon+\epsilon^{2}
\end{gathered}
$$

so that

$$
\lim _{\epsilon \downarrow 0} \frac{z_{0}(\epsilon)}{x_{0}(\epsilon)}=1,
$$

which indeed implies that $p^{*} \neq-d^{*}$, see Theorem 7 .

The third case is a problem where strong duality holds, but there exists no complementary solution pair (see Vandenberghe and Boyd [27]).

Example 3 (Strongly dual) Let $\bar{n}=2$ and

$$
B=\left[\begin{array}{ll}
0 & 0 \\
& 1
\end{array}\right], \quad C=\left[\begin{array}{ll}
0 & 1 \\
& 0
\end{array}\right], \quad \mathcal{A}=\left\{X \mid X=\left[\begin{array}{cc}
0 & x_{12} \\
& 0
\end{array}\right]\right\}
$$




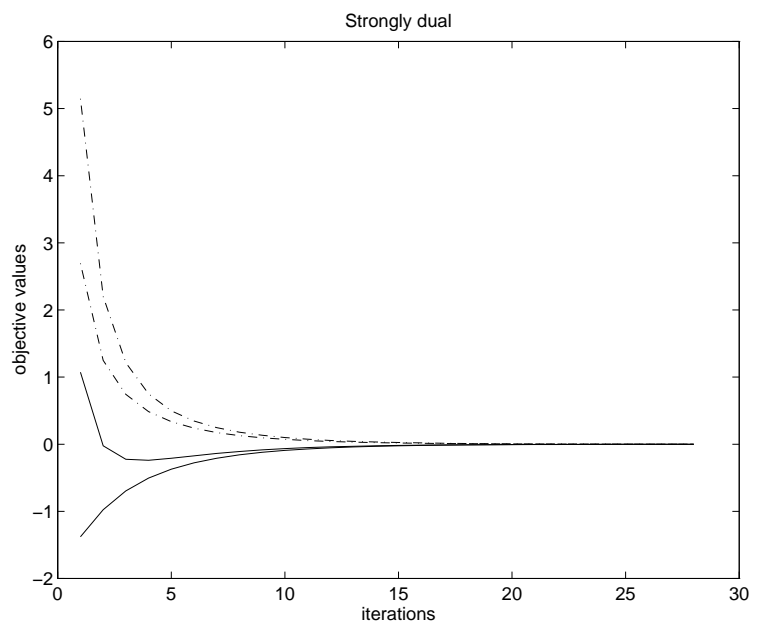

Figure 3: Example 3

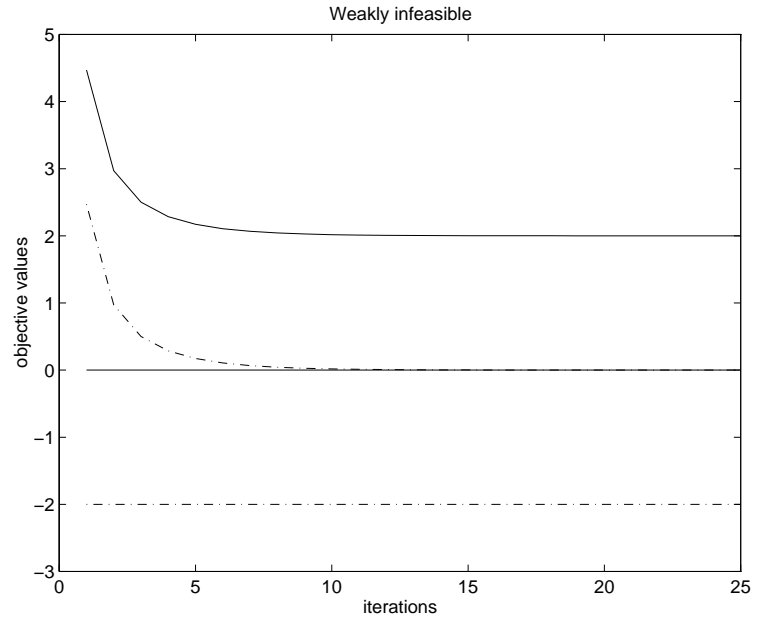

Figure 4: Example 4

so that the primal is solvable and weakly feasible,

$$
p^{*}=\inf \left\{2 x_{12} \mid X=\left[\begin{array}{cc}
0 & x_{12} \\
& 1
\end{array}\right] \succeq 0\right\}=0
$$

and the dual is strongly feasible but not solvable,

$$
d^{*}=\inf \left\{z_{22} \mid Z=\left[\begin{array}{cc}
z_{11} & 1 \\
& z_{22}
\end{array}\right] \succeq 0\right\}=0 .
$$

Notice that $p^{*}+d^{*}=0$, but the dual has no optimal solution. A weakly centered sequence for $0<\epsilon \leq 1 / 3$ is

$$
\begin{gathered}
X(\epsilon)=\epsilon^{3} I+\left[\begin{array}{rr}
0 & -\epsilon^{2} \\
\epsilon
\end{array}\right], \quad Z(\epsilon)=\epsilon^{3} I+\left[\begin{array}{cc}
3-\left(2 \epsilon+3 \epsilon^{2}+3 \epsilon^{3}\right) & \epsilon \\
& \epsilon^{2}
\end{array}\right], \\
y_{0}(\epsilon)=\epsilon^{3}, \quad x_{0}(\epsilon)=\epsilon+\epsilon^{3}, \quad z_{0}(\epsilon)=2 \epsilon^{2}+\epsilon^{3} .
\end{gathered}
$$

Hence,

$$
\lim _{\epsilon \downarrow 0} x_{0}(\epsilon)=0, \quad \lim _{\epsilon \downarrow 0} \frac{z_{0}(\epsilon)}{x_{0}(\epsilon)}=0,
$$

which indeed holds if and only if the primal is weakly feasible and the dual is not solvable, see Theorem 8.

So far, we have seen a weakly infeasible problem with $\lim _{\epsilon \downarrow 0} z_{0}(\epsilon) / x_{0}(\epsilon)=\infty$, a feasible problem with only weak duality and $\lim _{\epsilon \downarrow 0} z_{0}(\epsilon) / x_{0}(\epsilon) \in(0, \infty)$ and a strongly dual problem with 
$\lim _{\epsilon \downarrow 0} z_{0}(\epsilon) / x_{0}(\epsilon)=0$. The reader may wonder whether the asymptotic behavior of the indicator $z_{0}(\epsilon) / x_{0}(\epsilon)$ completely characterizes the three cases that we consider. Unfortunately, this is not the case, as the next example shows.

Example 4 (Weakly infeasible) Let $\bar{n}=2$, and consider

$$
B=\left[\begin{array}{ll}
0 & 1 \\
& 0
\end{array}\right], \quad C=0, \quad \mathcal{A}=\left\{X \mid X=\left[\begin{array}{cc}
0 & 0 \\
& x_{22}
\end{array}\right] \succeq 0\right\} .
$$

The primal is weakly infeasible,

$$
p^{*}=\inf \left\{0 \mid X=\left[\begin{array}{cc}
0 & 1 \\
& x_{22}
\end{array}\right] \succeq 0\right\}=\infty,
$$

and the dual is solvable and weakly feasible,

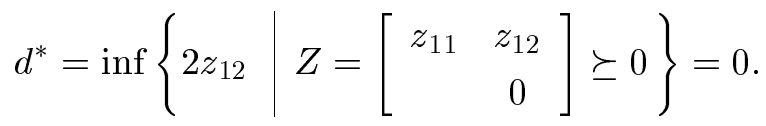

We construct a weakly centered sequence for $0<\epsilon \leq 1 / 3$ as

$$
\begin{gathered}
X(\epsilon)=\epsilon^{2} I+\left[\begin{array}{ll}
0 & \epsilon \\
& 1
\end{array}\right], \quad Z(\epsilon)=\epsilon^{2} I+\left[\begin{array}{cc}
2-3\left(\epsilon+\epsilon^{2}\right) & -\epsilon \\
& 0
\end{array}\right], \\
y_{0}(\epsilon)=\epsilon^{2}, \quad x_{0}(\epsilon)=\epsilon+\epsilon^{2}, \quad z_{0}(\epsilon)=2 \epsilon+\epsilon^{2} .
\end{gathered}
$$

We see that

$$
\lim _{\epsilon \downarrow 0} \frac{z_{0}(\epsilon)}{x_{0}(\epsilon)}=2
$$

The reader may still wonder whether we can distinguish weak infeasibility from strong duality. It appears somewhat difficult indeed, to construct an example of an infeasible problem where $z_{0}(\epsilon) / x_{0}(\epsilon) \rightarrow 0$, but it does exist.

Example 5 (Weakly infeasible) Let $\bar{n}=3$ and

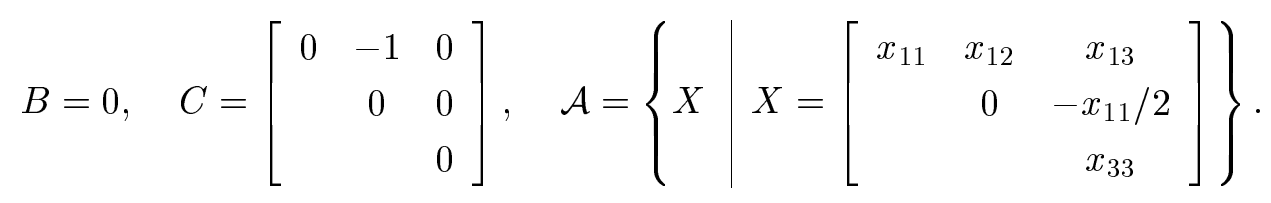

The primal is solvable and weakly feasible,

$$
p^{*}=\inf \left\{-2 x_{12} \mid X=\left[\begin{array}{ccc}
x_{11} & x_{12} & x_{13} \\
& 0 & -x_{11} / 2 \\
& & x_{33}
\end{array}\right] \succeq 0\right\}=0
$$




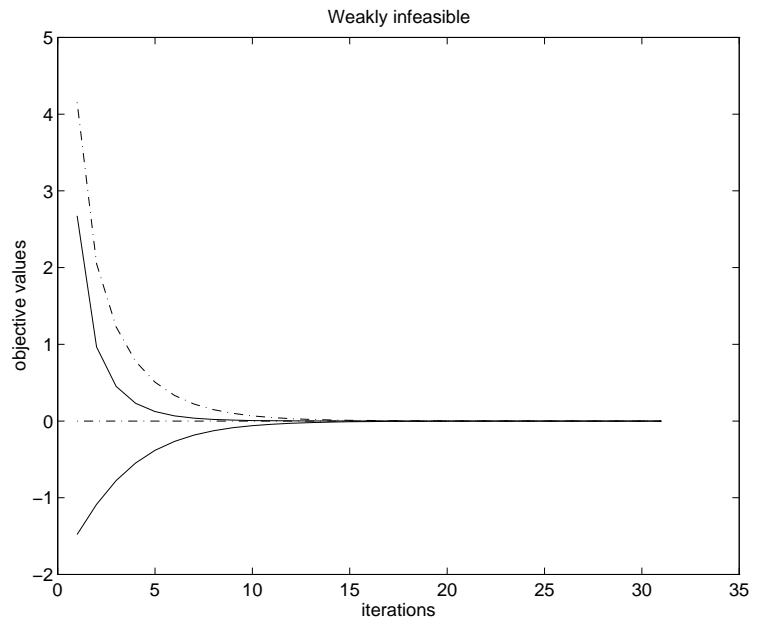

Figure 5: Example 5

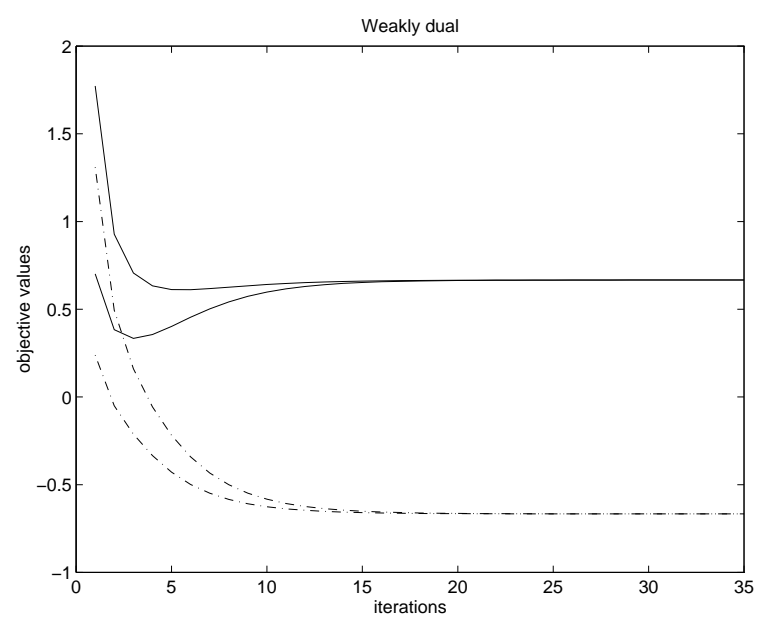

Figure 6: Example 6

and the dual is weakly infeasible,

$$
d^{*}=\inf \left\{0 \mid Z=\left[\begin{array}{ccc}
z_{11} & -1 & 0 \\
& z_{22} & z_{11} \\
& & 0
\end{array}\right] \succeq 0\right\}=\infty .
$$

We construct a weakly centered sequence for $0<\epsilon \leq 1 / 3$ by

$$
\begin{aligned}
& X(\epsilon)=\epsilon^{4} I+\left[\begin{array}{ccc}
\epsilon^{2} & \epsilon^{3} / 2 & 0 \\
& 0 & -\epsilon^{2} / 2 \\
& & 1
\end{array}\right] \\
& Z(\epsilon)=\epsilon^{4} I+\left[\begin{array}{ccc}
\epsilon^{2} & -\epsilon & 0 \\
& 3-\left(\epsilon+2 \epsilon^{2}+\epsilon^{3}+4 \epsilon^{4}\right) & \epsilon^{2} \\
& & 0
\end{array}\right] \text {, } \\
& y_{0}(\epsilon)=\epsilon^{4}, \quad x_{0}(\epsilon)=\epsilon+\epsilon^{4}, \quad z_{0}(\epsilon)=\epsilon^{3}+\epsilon^{4},
\end{aligned}
$$

so that

$$
\lim _{\epsilon \downarrow 0} \frac{z_{0}(\epsilon)}{x_{0}(\epsilon)}=0
$$

After Example 5 there is little hope that feasibility with lack of strong duality would imply $\lim \sup _{\epsilon \downarrow 0} z_{0}(\epsilon) / x_{0}(\epsilon)>0$. Indeed, we can construct a feasible problem with only weak duality but $\lim _{\epsilon \downarrow 0} z_{0}(\epsilon) / x_{0}(\epsilon)=0$. 
Example 6 (Weakly dual) Let $\bar{n}=4$ and

$$
\begin{gathered}
B=\left[\begin{array}{cccc}
0 & 1 / 3 & 0 & 0 \\
& 0 & 0 & 0 \\
& 0 & 0 \\
& & 1 / 3
\end{array}\right], \quad C=\left[\begin{array}{cccc}
0 & -1 / 3 & 0 & 0 \\
& 0 & 0 & 0 \\
& 0 & 0 \\
& & 2 / 3
\end{array}\right], \\
\mathcal{A}=\left\{X \mid X=\left[\begin{array}{cccc}
x_{11} & -x_{44} / 2 & x_{13} & x_{14} \\
& 0 & -x_{11} / 2 & x_{24} \\
& & x_{33} & x_{34} \\
& & & x_{44}
\end{array}\right]\right\}
\end{gathered}
$$

The primal is solvable and weakly feasible,

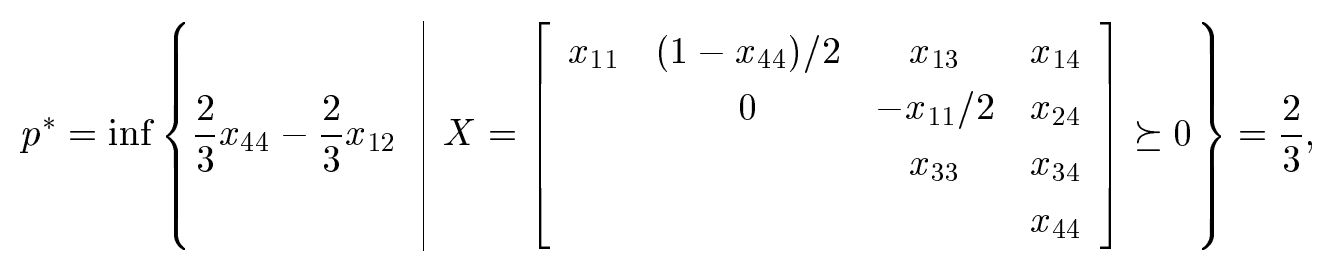

and the dual is also solvable and weakly feasible,

$$
d^{*}=\inf \left\{\frac{2}{3} z_{12}+\frac{1}{3} z_{44} \mid Z=\left[\begin{array}{cccc}
z_{11} & z_{44}-1 & 0 & 0 \\
& z_{22} & z_{11} & 0 \\
& & 0 & 0 \\
& & & z_{44}
\end{array}\right] \succeq 0\right\}=\frac{1}{3}
$$

Hence, $p^{*}+d^{*}=1>0$, so that strong duality fails. Remark that the first three rows and columns of this program are the same as in Example 5, with the additional constraints

$$
x_{12}=\frac{1-x_{44}}{2} \leq \frac{1}{2}, \quad z_{12}=z_{44}-1 \geq-1 .
$$

As a consequence, we can construct a weakly centered sequence that is very similar to the one in Example 5, viz.

$$
\begin{gathered}
X(\epsilon)=\epsilon^{4} I+\left[\begin{array}{cccc}
\epsilon^{2} & \epsilon^{3} / 2 & 0 & 0 \\
& 0 & -\epsilon^{2} / 2 & 0 \\
& 1 & 0 \\
& & \epsilon-\epsilon^{3}
\end{array}\right], \\
Z(\epsilon)=\epsilon^{4} I+\left[\begin{array}{cccc}
\epsilon^{2} & -\epsilon & 0 & 0 \\
& 4-\left(2 \epsilon+2 \epsilon^{2}+5 \epsilon^{4}\right) & \epsilon^{2} & 0 \\
& & 0 & 0 \\
& & & 0
\end{array}\right],
\end{gathered}
$$




\begin{tabular}{|l|ccc|}
\cline { 2 - 4 } \multicolumn{1}{c|}{} & \multicolumn{3}{c|}{$\lim \sup _{\epsilon \downarrow 0} z_{0}(\epsilon) / x_{0}(\epsilon)$} \\
\cline { 2 - 4 } \multicolumn{1}{c|}{} & 0 & $(0, \infty)$ & $\infty$ \\
\hline$p_{\mathrm{SD}}^{*}=\infty$ & Example 5 & Example 4 & Example 1 \\
$p_{\mathrm{SD}}^{*} \in(0, \infty)$ & Example 6 & Example 2 & impossible \\
$p_{\mathrm{SD}}^{*}=0$ & Example 3 & impossible & impossible \\
\hline
\end{tabular}

Table 1: Distinguishing the difficult cases where $x_{0}^{(k)}+s_{0}^{(k)} \rightarrow 0$

$$
y_{0}(\epsilon)=\epsilon^{4}, \quad x_{0}(\epsilon)=\epsilon+\epsilon^{4}, \quad z_{0}(\epsilon)=\epsilon^{3}+\epsilon^{4},
$$

and

$$
\lim _{\epsilon \downarrow 0} \frac{z_{0}(\epsilon)}{x_{0}(\epsilon)}=0
$$

Our results on the indicator

$$
\limsup _{\epsilon \downarrow 0} \frac{z_{0}(\epsilon)}{x_{0}(\epsilon)}
$$

are summarized in Table 1. The possible combinations in the table are illustrated by Examples 1-6. The impossibility of the remaining combinations follows from Theorem 5; see also Remark 4 and Remark 5.

As promised in Remark 8, we will now give an example where

$$
\lim _{\epsilon \downarrow 0} \frac{c^{\mathrm{T}} x(\epsilon)+s_{0}(\epsilon)}{x_{0}(\epsilon)}=-\infty,
$$

but $(\mathrm{P})$ is not unbounded. We consider this as an extremely nasty case, since it implies

$$
b^{\mathrm{T}} s(\epsilon) / x_{0}(\epsilon) \rightarrow \infty
$$

even though $d^{-}=-p^{*}<\infty$.

Example 7 We consider a semidefinite program, which has some similarity to Example 5, viz.

$$
p^{*}=\inf \left\{2 x_{45} \mid\left[\begin{array}{ccccc}
x_{11} & -x_{55} / 2 & x_{13} & & \\
& x_{22} & x_{11} & & \\
& & 0 & & \\
& & & 1 & x_{45} \\
& & & & x_{55}
\end{array}\right] \succeq 0\right\}=0,
$$

for which the dual is weakly infeasible,

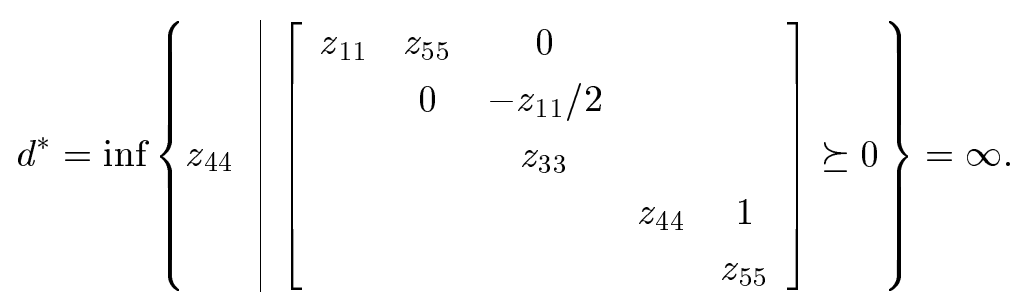




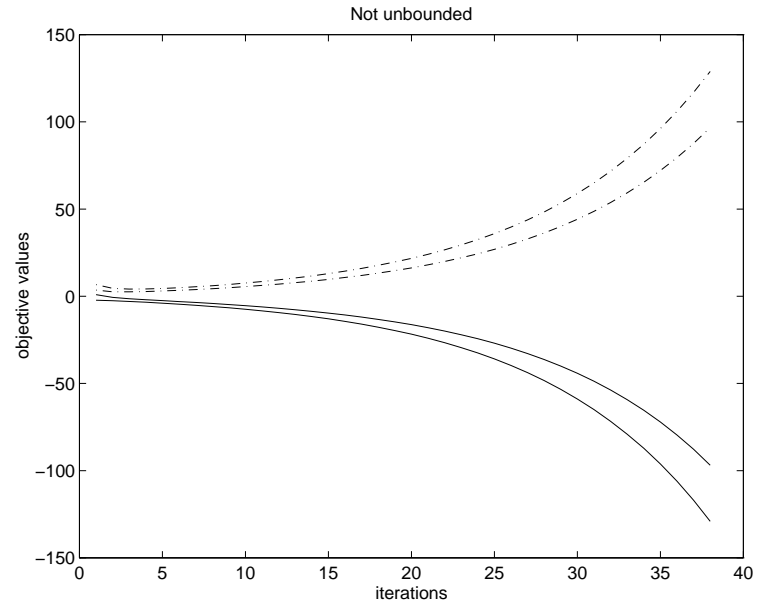

Figure 7: Example 7

Notice however, that $d^{-}=0$. We construct a weakly centered sequence for small $\epsilon$ by

$$
\begin{aligned}
& X(\epsilon)=\epsilon^{12} I+\left[\begin{array}{ccccc}
\epsilon^{6} & -\epsilon^{3} / 2 & 0 & \\
& 3-O\left(\epsilon^{3}\right) & \epsilon^{6} & & \\
& & \epsilon^{12} & & \\
& & & \epsilon^{7} & -\epsilon^{5} \\
& & & \epsilon^{3}
\end{array}\right], \\
& Z(\epsilon)=\epsilon^{12} I+\left[\begin{array}{ccccc}
\epsilon^{6} & \epsilon^{9} & 0 & & \\
& \epsilon^{12} & -\epsilon^{6} / 2 & \\
& 3-O\left(\epsilon^{3}\right) & \\
& & & \epsilon^{5} & \epsilon^{7} \\
& & & \epsilon^{9}
\end{array}\right], \\
& y_{0}(\epsilon)=\epsilon^{12}, \quad x_{0}(\epsilon)=\epsilon^{7}+\epsilon^{12}, \quad z_{0}(\epsilon)=\epsilon^{5}+\epsilon^{12} .
\end{aligned}
$$

We have

$$
\lim _{\epsilon \downarrow 0} \frac{c^{T} x(\epsilon)+z_{0}(\epsilon)}{x_{0}(\epsilon)}=\frac{-2 \epsilon^{5}+\epsilon^{5}+\epsilon^{12}}{\epsilon^{7}+\epsilon^{12}}=-\infty,
$$

but $(P)$ is not unbounded. See Corollary 1 and Remark 8.

\section{$7 \quad$ Existence of Weakly Centered Sequences}

In this section, we will prove the existence of a weakly centered sequence for ( $\mathrm{E}$ ), if we use the primal-dual model $\mathrm{CP}\left(b_{\mathrm{SD}}, c_{\mathrm{SD}}, \mathcal{A}_{\mathrm{SD}}, \mathcal{K}_{\mathrm{SD}}\right)$ of Section 6 . In fact, we will give a constructive proof 
using the theory of logarithmically homogeneous barriers, which has been developed by Nesterov and Nemirovsky [16].

Definition 3 Let $\mathcal{K}$ be a closed, solid and pointed convex cone. Then $F$ : int $\mathcal{K} \rightarrow \Re$ is a $\nu$ logarithmically homogeneous barrier for $\mathcal{K}$ if $F$ is a twice continuously differentiable convex function on int $\mathcal{K}$ such that $F\left(x^{(i)}\right) \rightarrow \infty$ for any sequence $x^{(i)}$ in int $\mathcal{K}, i=1,2, \ldots$, that converges to the boundary of $\mathcal{K}$, and

$$
F(t x)=F(x)-\nu \log t \text { for all } x \in \text { int } \mathcal{K}, t>0,
$$

where $\nu \geq 1$ is a fixed parameter.

It is known that any closed, pointed and solid convex cone $\mathcal{K}$ is endowed with a logarithmically homogeneous barrier, see Theorem 2.5.1 in [16]. Moreover, Proposition 2.3.5 in [16] states that logarithmically homogeneous barriers are strictly convex functions. Important special cases are the $n$-logarithmically homogeneous barrier $F(x)=-\sum_{i=1}^{n} \log x_{i}$ for the cone $\mathcal{K}=\Re_{+}^{n}$, and the $n$-logarithmically homogeneous barrier $F(X)=-\log \operatorname{det} X$ for the cone $\mathcal{K}=\mathcal{H}_{+}^{(n)}$.

Let $F(x)$ be a $\nu$-logarithmically homogeneous barrier for $\mathcal{K}$, and define its conjugate (or LegendreYoung-Fenchel transform) by

$$
F^{*}(z):=\sup _{x \in \operatorname{int} \mathcal{K}}\left\{(-z)^{\mathrm{T}} x-F(x)\right\} .
$$

Notice that if $z \notin \mathcal{K}^{*}$, then $(-z)^{\mathrm{T}} x>0$ for some $x \in$ int $\mathcal{K}$, which together with (26) implies $F^{*}(z)=\infty$. In fact, Nesterov and Nemirovsky [16] showed that $F^{*}(z)$ is a $\nu$-logarithmically homogeneous barrier for $\mathcal{K}^{*}$ (see Theorem 2.4.4 therein). Hence, $F^{*}(z)<\infty$ if and only if $z \in$ int $\mathcal{K}^{*}$. Moreover, the biconjugate $F^{* *}$ of $F$ is again $F$, i.e. $F(x)=F^{* *}(x)$, see e.g. Rockafellar [22]. Let $x \in$ int $\mathcal{K}$. Using definition $(27)$ and the first order optimality conditions for concave maximization, it follows that

$$
F^{*}(z)=(-z)^{\mathrm{T}} x-F(x) \text { if } z=-\nabla F(x)
$$

from which we obtain

$$
-\nabla F(x) \in \operatorname{int} \mathcal{K}^{*}
$$

and

$$
\nabla F^{*}(z)=-x \text { if } z=-\nabla F(x) .
$$

Using (26), it is straightforward to show that

$$
\nabla F(x / t)=t \nabla F(x) \text { for all } t>0 \text {, }
$$

and

$$
\nabla F(x)^{\mathrm{T}} x=-\nu .
$$


The above properties of $F$ are also listed by Nesterov and Todd [17] and Nesterov, Todd and Ye [19]. Based on the barrier $F$, one can define a barrier path with parameter $\mu>0$, see Theorem 9 below. In the case of semidefinite programming, the central path is the barrier path for $F(x)=-\log (\operatorname{det} X)$.

Theorem 9 (barrier path) Let $\mathcal{K}$ be a convex cone that is closed, pointed and solid. Suppose that $C P(b, c, \mathcal{A}, \mathcal{K})$ is a conic convex program that is primal and dual strongly feasible. Let $F:$ int $\mathcal{K} \rightarrow \Re$ be a $\nu$-logarithmically homogeneous barrier for $\mathcal{K}$, and define

$$
\phi_{\mu}(x):=(-c)^{T} x-\mu F(x)
$$

with $\mu>0$. Then there exists a unique vector $x(\mu) \in(b+\mathcal{A}) \cap$ int $\mathcal{K}$ such that

$$
\phi_{\mu}(x(\mu))=\max \left\{\phi_{\mu}(x) \mid x \in(b+\mathcal{A}) \cap \operatorname{int} \mathcal{K}\right\} .
$$

Moreover, letting $z(\mu):=-\mu \nabla F(x(\mu))$, there holds

$$
x(\mu)^{T} z(\mu)=\nu \mu, \quad x(\mu)=-\mu \nabla F^{*}(z(\mu)),
$$

and

$$
z(\mu)=\arg \max \left\{(-b)^{T} z-\mu F^{*}(z) \mid z \in\left(c+\mathcal{A}^{\perp}\right) \cap \text { int } \mathcal{K}^{*}\right\} .
$$

Proof. First, notice that $\phi_{\mu}(\cdot)$ is a concave function. Moreover, for all $x \in(b+\mathcal{A}) \cap$ int $\mathcal{K}$ and $z \in\left(c+\mathcal{A}^{\perp}\right) \cap \operatorname{int} \mathcal{K}^{*}$, there holds

$$
\phi_{\mu}(x)=b^{\mathrm{T}} z-z^{\mathrm{T}} x-\mu F(x) \leq b^{\mathrm{T}} z+\mu F^{*}(z / \mu) .
$$

Hence, $\phi_{\mu}(x)$ is bounded from above on $(b+\mathcal{A}) \cap$ int $\mathcal{K}$, and since $\phi_{\mu}(\cdot)$ is a strictly concave function, it follows that $\phi_{\mu}(\cdot)$ achieves a maximum, and the maximizer $x(\mu)$ is unique. From the first-order optimality conditions, we know that $x(\mu)$ satisfies

$$
\nabla \phi_{\mu}(x(\mu))=-c-\mu \nabla F(x(\mu)) \in \mathcal{A}^{\perp} .
$$

Letting $z(\mu):=-\mu \nabla F(x(\mu))$, it follows from (28) that $z(\mu) \in\left(c+\mathcal{A}^{\perp}\right) \cap$ int $\mathcal{K}^{*}$, and using (29)-(30), we have

$$
x(\mu)=-\nabla F^{*}(z(\mu) / \mu)=-\mu \nabla F^{*}(z(\mu)),
$$

so that

$$
-b-\mu \nabla F^{*}(z(\mu)) \in \mathcal{A} .
$$

The above relation shows that $z(\mu)$ satisfies the optimality conditions for

$$
\max \left\{(-b)^{\mathrm{T}} z-\mu F^{*}(z) \mid z \in\left(c+\mathcal{A}^{\perp}\right) \cap \text { int } \mathcal{K}^{*}\right\} .
$$


Finally, it follows from (31) that

$$
z(\mu)^{\mathrm{T}} x(\mu)=-\mu \nabla F(x(\mu))^{\mathrm{T}} x(\mu)=\nu \mu .
$$

We now propose the following barrier for $\mathcal{K} \times \mathcal{K}^{*}$,

$$
F_{\mathrm{SD}}(x, z):=F(x)+F^{*}(z) .
$$

Using (26), we see that $F_{\mathrm{SD}}(x, z)$ is a $2 \nu$-logarithmically homogeneous barrier, and from definition (27) and the fact that $F(x)=F^{* *}(x)$, we obtain that

$$
F_{\mathrm{SD}}^{*}(z, x)=F_{\mathrm{SD}}(x, z)
$$

This leads to the following definition:

Definition 4 Let $\mathcal{K}$ be a solid convex cone such that $\mathcal{K}^{*}=\Pi \mathcal{K}$ for some symmetric permutation matrix $\Pi$. A $\nu$-logarithmically homogeneous barrier $F:$ int $\mathcal{K} \rightarrow \Re$ for $\mathcal{K}$ is $\Pi$ self-conjugate if and only if

$$
F^{*}(\Pi x)=F(x) \text { for all } x \in \operatorname{int} \mathcal{K} \text {. }
$$

For the extended self-dual model (E), we define

$$
F_{\mathrm{E}}\left(x_{\mathrm{SD}}, x_{0}, z_{0}\right):=F_{\mathrm{SD}}\left(x_{\mathrm{SD}}\right)-\log x_{0}-\log z_{0}
$$

where $F_{\mathrm{SD}}$ is a $(2 \nu)$-logarithmically homogeneous $\Pi_{\mathrm{SD}}$ self-conjugate barrier for $\mathcal{K}_{\mathrm{SD}}$. It is easy to verify that $F_{\mathrm{E}}$ is then a $(2 \nu+2)$-logarithmically homogeneous $\Pi_{\mathrm{H}}$ self-conjugate barrier for $\mathcal{K}_{\mathrm{H}}$. Since (E) is strongly feasible (see Theorem 3), we can apply Theorem 9 to arrive at the following result.

Theorem 10 Define

$$
\phi_{\mu}\left(x_{E}\right):=\left(-c_{E}\right)^{T} x-\mu F_{E}\left(x_{E}\right),
$$

and let

$$
x_{E}(\mu):=\arg \max \left\{\phi_{\mu}\left(x_{E}\right) \mid x_{E} \in\left(b_{E}+\mathcal{A}_{E}\right) \cap \operatorname{int} \mathcal{K}_{E}\right\}
$$

Then

$$
x_{0}(\mu) z_{0}(\mu)=\mu=\frac{1}{\nu+1} c_{E}^{T} x_{E}(\mu)
$$

and $\lim _{\mu \downarrow 0} c_{E}^{T} x_{E}(\mu)=0$. 
Proof. From Theorem 9, we know that $x_{\mathrm{E}}(\mu)$ is well defined, and

$$
x_{\mathrm{E}}(\mu)^{\mathrm{T}} z_{\mathrm{E}}(\mu)=2(\nu+1) \mu, \quad x_{\mathrm{E}}(\mu)=-\mu \nabla F_{\mathrm{E}}^{*}\left(z_{\mathrm{E}}(\mu)\right),
$$

where $z_{\mathrm{E}}(\mu):=-\mu \nabla F_{\mathrm{E}}\left(x_{\mathrm{E}}(\mu)\right)$. In addition, Theorem 9 tells us that $z_{\mathrm{E}}(\mu)$ is the maximizer of the function $-b_{\mathrm{E}}^{\mathrm{T}} z_{\mathrm{E}}-\mu F_{\mathrm{E}}^{*}\left(z_{\mathrm{E}}\right)=\phi_{\mu}\left(\Pi_{\mathrm{H}} z_{\mathrm{E}}\right)$ over all dual interior solutions $z_{\mathrm{E}}$, and therefore

$$
x_{\mathrm{E}}(\mu)=\Pi_{\mathrm{H}} z_{\mathrm{E}}(\mu)=-\mu \Pi_{\mathrm{H}} \nabla F_{\mathrm{E}}\left(x_{\mathrm{E}}(\mu)\right) .
$$

Noticing that

$$
\nabla F_{\mathrm{E}}\left(x_{\mathrm{E}}\right)=\left[\nabla F_{\mathrm{SD}}\left(x_{\mathrm{SD}}\right)^{\mathrm{T}}, \quad-1 / x_{0},-1 / z_{0}\right]^{\mathrm{T}},
$$

we obtain from (32) that

$$
x_{0}(\mu)=\mu / z_{0}(\mu) .
$$

Hence,

$$
x_{0}(\mu) z_{0}(\mu)=\mu=\frac{1}{2(\nu+1)} x_{\mathrm{E}}(\mu)^{\mathrm{T}} z_{\mathrm{E}}(\mu)=\frac{1}{\nu+1} c_{\mathrm{E}}^{\mathrm{T}} x_{\mathrm{E}}(\mu),
$$

where we used Theorem 1 .

Theorem 10 shows that $\left\{x_{\mathrm{E}}(\mu) \mid \mu>0\right\}$ is a weakly centered sequence for (E).

We have used the theory of logarithmically homogeneous barriers, to establish the existence of weakly centered sequences for conic convex programming. In the special case of semidefinite programming however, there is no need for this barrier argument. Namely, consider the primal-dual path-following methods such as treated in e.g. [8, 9, 15, 17, 24, 23]. We may initialize these methods with a primal-dual pair $\left(x_{\mathrm{E}}^{(0)}, z_{\mathrm{E}}^{(0)}\right)$ that satisfies $z_{\mathrm{E}}^{(0)}=\Pi_{\mathrm{H}} x_{\mathrm{E}}^{(0)}$, i.e. the initial primal and dual solutions are essentially the same. In particular, we may start with the identity solution, as explained in Section 6.1. It is then easily checked that all subsequent iterates $\left(x_{\mathrm{E}}^{(k)}, z_{\mathrm{E}}^{(k)}\right)$ also satisfy such a property. Namely, primal-dual path-following algorithms generate iterates in a so-called $\mathcal{N}_{\infty}^{-}(\beta)$-neighborhood of the central path,

which by definition implies that

$$
x_{0}^{(k)} z_{0}^{(k)} \geq(1-\beta) \frac{\left(x_{\mathrm{E}}^{(k)}\right)^{\mathrm{T}} z_{\mathrm{E}}^{(k)}}{2 n+2} .
$$

This shows that iterative sequences in the $\mathcal{N}_{\infty}^{-}(\beta)$-neighborhood are weakly centered with $\omega=$ $(1-\beta) /(n+1)$. 


\section{Conclusions}

In this paper, we addressed questions such as how to solve a general conic convex program and certify the solution(s) obtained. It turned out that a conic convex program can be in one or more of the following states: 1) It is solvable; 2) It is weakly infeasible; 3) It is strongly infeasible; 4) It is weakly feasible; and 5) It is strongly feasible. Certificates verifying a given state involve the dual solutions. To get a complete picture about the problem, we need to solve a primal-dual embedded system, for which we studied relevant properties. As a natural next step, we showed how the selfdual embedding technique [30] for linear programming can be extended to this general case. By a central path following method, we further proved that a weakly centered sequence for the self-dual embedding system will be generated, which indeed provides much information about the solution to the original problem. By various examples from semidefinite programming, we demonstrated several intricate cases which can never occur in linear programming. We conclude from this study that solving a general conic convex program requires substantially more effort and care than solving a classical linear programming problem. Nevertheless, it is also clear from our study that the selfdual embedding technique and the path-following methodology provide good tools for solving conic convex programming problems, as long as an easy computable self-concordant barrier of the cone is available.

We remark that similar techniques can be used to deal with the strict feasibility problem:

$$
\text { Find } x \in(b+\mathcal{A}) \cap \text { int } \mathcal{K} \text {. }
$$

This includes the problem of finding a matrix satisfying a set of matrix inequalities strictly; such problems arise in stability analysis for linear differential inclusions [1]. If we use an algorithm that produces a maximally complementary solution, such as the path-following algorithm for semidefinite programming [6], then the self-dual embedding technique can be applied directly on (P) with $c=0$, to solve the strict feasibility problem. But if the algorithm generates merely weakly centered sequences, then we should apply the embedding technique to the following, auxiliary problem:

Find $x, t$ such that $x-\iota \in \mathcal{K}$ and $x-t b \in \mathcal{A}$,

where $\iota$ is a given vector in the interior of $\mathcal{K}$. If (33) has a solution, then it is even possible to find it in finite time (in the real number computational model). Otherwise, i.e. if (33) has no solution, then we obtain in the limit a certificate $z$ satisfying

$$
\iota^{\mathrm{T}} z<0, b^{\mathrm{T}} z=0, z \in \mathcal{A}^{\perp} \cap \mathcal{K}^{*}
$$

which indeed demonstrates infeasibility of (33). 


\section{References}

[1] S. Boyd, L. El Ghaoui, E. Feron, and V. Balakrishnan. Linear matrix inequalities in system and control theory, volume 15 of Studies in Applied Mathematics. SIAM, Philadelphia, PA, 1994.

[2] R.J. Duffin. Infinite programs. In H.W. Kuhn and A.W. Tucker, editors, Linear Inequalities and Related Systems, pages 157-170. Princeton University Press, Princeton, NJ, 1956.

[3] D.M. Gay. Electronic mail distribution of linear programming test problems. Mathematical Programming Society Committee on Algorithms News Letter, 13:10-12, 1985.

[4] A.J. Goldman and A.W. Tucker. Polyhedral convex cones. In H.W. Kuhn and A.W. Tucker, editors, Linear Inequalities and Related Systems, pages 19-40. Princeton University Press, Princeton, NJ, 1956.

[5] O. Güler and Y. Ye. Convergence behavior of interior-point algorithms. Mathematical Programming, 60:215-228, 1993.

[6] E. de Klerk, C. Roos, and T. Terlaky. Initialization in semidefinite programming via a self-dual skew-symmetric embedding. Technical Report 96-10, Delft University of Technology, Faculty of Technical Mathematics and Informatics, Delft, The Netherlands, 1996.

[7] E. de Klerk, C. Roos, and T. Terlaky. Infeasible start semidefinite programming algorithms via self-dual embeddings. Technical Report 97-10, Delft University of Technology, Faculty of Technical Mathematics and Informatics, Delft, The Netherlands, 1997.

[8] M. Kojima, S. Shindoh, and S. Hara. Interior-point methods for the monotone semidefinite linear complementarity problem in symmetric matrices. SIAM Journal on Optimization, $7(1): 86-125,1997$.

[9] C.J. Lin and R. Saigal. An infeasible start predictor corrector method for semi-definite linear programming. Technical report, Department of Industrial and Operations Engineering, The University of Michigan, Ann Arbor, USA, 1995.

[10] Z.-Q. Luo, J.F. Sturm, and S. Zhang. Duality and self-duality for conic convex programming. Technical Report 9620/A, Econometric Institute, Erasmus University Rotterdam, Rotterdam, The Netherlands, 1996.

[11] Z.-Q. Luo, J.F. Sturm, and S. Zhang. Duality results for conic convex programming. Technical Report 9719/A, Econometric Institute, Erasmus University Rotterdam, Rotterdam, The Netherlands, 1997. 
[12] I.J. Lustig, R.E. Marsten, and D.F. Shanno. Computational experience with a primal-dual interior point method for linear programming. Linear Algebra and its Applications, 152:191$222,1991$.

[13] I.J. Lustig, R.E. Marsten, and D.F. Shanno. On implementing Mehrotra's predictor-corrector interior point method for linear programming. SIAM Journal on Optimization, 2:435-449, 1992.

[14] S. Mizuno. Infeasible-interior-point algorithms. In T. Terlaky, editor, Interior-Point Methods of Mathematical Programming, pages 159-187. Kluwer Adademic Publishers, Dordrecht, 1996.

[15] R.D.C. Monteiro. Primal-dual path following algorithms for semidefinite programming. Technical report, School of Industrial and Systems Engineering, Georgia Tech, Atlanta, Georgia, U.S.A., 1995. To appear in SIAM Journal on Optimization 7 (1997) 3.

[16] Y. Nesterov and A. Nemirovsky. Interior point polynomial methods in convex programming, volume 13 of Studies in Applied Mathematics. SIAM, Philadelphia, 1994.

[17] Y. Nesterov and M.J. Todd. Primal-dual interior-point methods for self-scaled cones. Technical Report 1125, School of Operations Research and Industrial Engineering, Cornell University, Ithaca, New York, 1995.

[18] Y. Nesterov and M.J. Todd. Self-scaled barriers and interior-point methods for convex programming. Mathematics of Operations Research, 22(1):1-42, 1997.

[19] Y. Nesterov, M.J. Todd, and Y. Ye. Infeasible-start primal-dual methods and infeasibility detectors for nonlinear programming problems. Technical Report 1156, School of Operations Research and Industrial Engineering, Cornell University, Ithaca, New York, 1996.

[20] F.A. Potra and R. Sheng. Homogeneous interior-point algorithms for semidefinite programming. Technical report, Department of Mathematics, University of IOWA, Iowa City, IA, USA, 1995.

[21] M.V. Ramana. An exact duality theory for semidefinite programming and its complexity implications. Mathematical Programming, 77(2):129-162, 1997.

[22] R.T. Rockafellar. Convex Analysis. Princeton University Press, Princeton, NJ, 1970.

[23] J.F. Sturm. Primal-Dual Interior Point Approach to Semidefinite Programming, volume 156 of Tinbergen Institute Research Series. Thesis Publishers, Amsterdam, The Netherlands, 1997.

[24] J.F. Sturm and S. Zhang. Symmetric primal-dual path following algorithms for semidefinite programming. Technical Report 9554/A, Econometric Institute, Erasmus University Rotterdam, Rotterdam, The Netherlands, 1995. 
[25] M.J. Todd and Y. Ye. Approximate Farkas lemmas and stopping rules for iterative infeasiblepoint algorithms for linear programming. Technical report, School of Operations Research and Industrial Engineering, Cornell University, Ithaca, NY, USA, 1994.

[26] A.W. Tucker. Dual systems of homogeneous linear relations. In H.W. Kuhn and A.W. Tucker, editors, Linear Inequalities and Related Systems, pages 3-18. Princeton University Press, Princeton, NJ, 1956.

[27] L. Vandenberghe and S. Boyd. Semidefinite programming. SIAM Review, 38:49-95, 1996.

[28] X. Xu, P. Hung, and Y. Ye. A simplified homogeneous and self-dual linear programming algorithm and its implementation. Annals of Operations Research, 62:151-172, 1996.

[29] Y. Ye. On homogeneous and self-dual algorithms for LCP. Mathematical Programming, 76(1):211-221, 1997.

[30] Y. Ye, M.J. Todd, and S. Mizuno. An $O(\sqrt{n} L)$-iteration homogeneous and self-dual linear programming algorithm. Mathematics of Operations Research, 19:53-67, 1994. 